\title{
Experimental and computational micro-mechanical investigations of compressive properties of polypropylene/multi-walled carbon nanotubes nanocomposite foams
}

\author{
F. Wan ${ }^{\mathrm{a}, \mathrm{d}}$, M.-P. Tran ${ }^{\mathrm{b}}$, C. Leblanc ${ }^{\mathrm{c}}$, E. Béchet $^{\mathrm{c}}$, E. Plougonven $^{\mathrm{e}}, \mathrm{A}$.

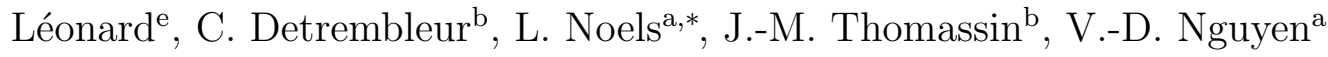 \\ ${ }^{a}$ University of Liege (ULg), Department of Aerospace and Mechanical Engineering, \\ Computational \& Multiscale Mechanics of Materials (CM3) \\ Quartier Polytech 1, Alle de la Dcouverte 9, B-4000 Liège, Belgium \\ ${ }^{b}$ University of Liege (ULg), \\ Center for Education and Research on Macromolecules (CERM), Chemistry Department, \\ Allée du 6 Août 13, B-4000 Liège, Belgium \\ ${ }^{c}$ University of Liege (ULg), Department of Aerospace and Mechanical Engineering, \\ Conception géométrique assistée par ordinateur \\ Quartier Polytech 1, Alle de la Dcouverte 9, B-4000 Liège, Belgium \\ ${ }^{d}$ Northwestern Polytechnical University (NPU), School of Aeronautics, \\ Xian 710072, China \\ ${ }^{e}$ University of Liege (ULg), \\ Chemical Engineering Lab, Department of Applied Chemistry \\ Allée du 6 Août 13, B-4000 Liège, Belgium
}

\begin{abstract}
The compressive behavior of nanocomposite foams is studied by both experimental and computational micro-mechanics approaches with the aim of providing an efficient computational model for this kind of material.

The nanocomposites based on polypropylene (PP) and different contents of multi-walled carbon nanotubes (CNTs) are prepared by melt mixing

*Corresponding author; Phone: +32 4366 4826; Fax: +32 43669505

Email addresses: fwan@nwpu.edu.cn (F. Wan), azianphuong@yahoo.com (M.-P. Tran), christophe.leblanc@ulg.ac.be (C. Leblanc), eric.bechet@ulg.ac.be (E. Béchet), eplougonven@ulg.ac.be (E. Plougonven), A.Leonard@ulg.ac.be (A.

Léonard), christophe.detrembleur@ulg.ac.be (C. Detrembleur), L. Noels@ulg.ac.be (L. Noels), jm.thomassin@ulg.ac.be (J.-M. Thomassin), vandung.nguyen@ulg.ac.be (V.-D. Nguyen)
\end{abstract}


method. The nanocomposite samples are foamed using super-critical carbon dioxide $\left(\mathrm{ScCO}_{2}\right)$ as blowing agent at different soaking temperatures. The influence of this foaming parameter on the morphological characteristics of the foam micro-structure is discussed. Differential Scanning Calorimetry (DSC) measurements are used to quantify the crystallinity degree of both nanocomposites and foams showing that the crystallinity degree is reduced after the foaming process. This modification leads to mechanical properties of the foam cell walls that are different from the raw nanocomposite PP/CNTs material. Three-point bending tests are performed on the latter to measure the flexural modulus in terms of the crystallinity degree. Uniaxial compression tests are then performed on the foamed samples under quasi-static conditions in order to extract the macro-scale compressive response.

Next, a two-level multi-scale approach is developed to model the behavior of the foamed nanocomposite material. On the one hand, the micromechanical properties of nanocomposite PP/CNTs cell walls are evaluated from a theoretical homogenization model accounting for the micro-structure of the semi-crystalline PP, for the degree of crystallinity, and for the CNT volume fraction. The applicability of this theoretical model is demonstrated via the comparison with experimental data from the described experimental measurements and from literature. On the other hand, the macroscopic behavior of the foamed material is evaluated using a computational micromechanics model using tetrakaidecahedron unit cells and periodic boundary conditions to estimate the homogenized properties. The unit cell is combined with several geometrical imperfections in order to capture the elastic collapse of the foamed material. The numerical results are compared to the experimental measurements and it is shown that the proposed unit cell computational micro-mechanics model can be used to estimate the homogenized behavior, including the linear and plateau regimes, of nanocomposite foams.

Keywords: Polypropylene, multi-walled carbon nanotubes, Nanocomposite foams, Computational micro-mechanics, Homogenization

\section{Introduction}

Recently, there has been a growing interest in artificially synthesized micro-structured materials. For instance, polymer nanocomposite foams, which are produced by foaming polymer nanocomposites, have received an increasing attention from both the scientific and industrial communities (Lee 
et al., 2005; Ibeh and Bubacz, 2008, e.g.). A polymer nanocomposite results from the dispersion of nano-fillers (with a size order of few nanometers in at least one dimension) in a polymer matrix. The combination of nanofillers, low density polymers, and foaming technology generates a new class of foamed materials which possess high specific and multi-functional characteristics. Their applications span almost all areas of research and industrial fields such as aerospace, automobile, packaging, electromagnetic interference (EMI) shielding (Thomassin et al., 2010), etc.

Since carbon nanotubes (CNTs) can exhibit exceptional properties such as extremely high strength, high stiffness, high aspect ratio, and high thermal and electrical conductivity, they are commonly used as reinforcing agents in polymer matrices (Spitalsky et al., 2010). Among the various existing polymers, polypropylene (PP) is a good compromise between properties, cost, and easiness of processability, making it a popular choice as the matrix phase (Prashantha et al., 2009; McIntosh et al., 2006; Jose et al., 2007; Xia et al., 2004; Kang et al., 2010; Manchado et al., 2005; Dondero and Gorga, 2006, among others). The properties of the resulting PP/CNTs nanocomposites depend not only on the properties of the nano-fillers (e.g. single-wall CNTs, double-wall CNTs, multi-walled CNTs), of the PP (e.g. polymer structure, crystallinity level), and on their percentages, but also on the processing methods as discussed by Spitalsky et al. (2010). Moreover, Chen et al. (2011) have shown that the mechanical properties of foamed nanocomposites are also strongly influenced by the morphological characteristics of their microstructure, such as cell density, cell size, and cell size distribution. As a result, the combination of the polymer nanocomposites and foaming technologies increases the material tailoring possibilities for lightweight applications.

$\mathrm{PP} / \mathrm{CNTs}$ nanocomposite foams of high volume expansions (larger than 10) possess several advantages as compared to other polymer-based foams, such as their relative low manufacturing cost and their light weight. Although the electromagnetic properties of such foams have already been studied (Thomassin et al., 2010; Ameli et al., 2014), their mechanical properties have not been carefully studied. The knowledge of those mechanical properties remains essential in most applications, and this is even truer for the studied PP/CNTs nanocomposite foams as the high volume expansion yields a rather low stiffness and makes them prone to buckling. However, nowadays it is in most cases difficult to predict the equivalent properties of these complex micro-structured materials without expensive and time-consuming experiments, which in turn, constrains material tailoring to trial and error 
tests. Therefore, numerical simulation techniques with high computational efficiency are necessary so that the problem of sensitivity analysis to the parameters of the micro-structure can be addressed, thus providing an optimization tool to carry out material tailoring. The aim of this work is to provide an efficient methodology to predict the compressive responses of $\mathrm{PP} / \mathrm{CNTs}$ nanocomposite foams with high volume expansions. In particular a numerical model is developed to predict, at first, their linear elastic response and, then, the plateau behavior under finite compressive strains arising from the cell-wall elastic buckling. This numerical model is informed and validated through experimental data.

To this end, PP/CNTs nanocomposite foams with $4 \mathrm{wt} \%$ and 8 wt\% multi-walled CNTs are studied. The PP matrix is a semi-crystalline polymer whose crystallinity can be modified during the foaming process according to the heat treatment, absorbed amount of blowing agent (Takada et al., 2001), or nano-fillers properties (Leelapornpisit et al., 2005). Super-critical carbon dioxide $\left(\mathrm{ScCO}_{2}\right)$ is used as blowing agent during the one-step batch foaming process. The crystallized state of the PP matrix is inevitably changed for the different nanocomposite preparations and foaming conditions, leading to the modification of its mechanical properties. This point is demonstrated through the results of Differential Scanning Calorimetry (DSC) of both unfoamed and foamed materials. In order to measure the material properties, three-point bending tests were performed on PP/CNTs nanocomposite (unfoamed) samples. Besides, measurements of the elastic flexural modulus and uniaxial compression tests were performed on the nanocomposite foamed samples. These tests clearly show the dependency of the material properties on the crystallinity degree.

The considered nanocomposite foams have a multi-scale behavior in nature, as shown in Fig. 1. At least three distinct scales can be considered: the macro-scale, the micro-scale, and the nano-scale. The macro-scale is characterized by a length $L_{\text {macro }}$ at the macro-structural level. The microscale is characterized by a length $L_{\text {micro }}$ of the order of several cells size and gives an insight to the foam micro-structure with its morphological characteristics, such as the cell size, the cell distribution, the cell shape, the thickness of the cell walls, etc. The nano-scale is characterized by a length $L_{\text {nano }}$ and describes the heterogeneity details of the nanocomposite material constituting the cell walls: the nano-fillers, their orientation, the amorphous or crystallized regions of the PP matrix. The multi-scale strategy adopted 


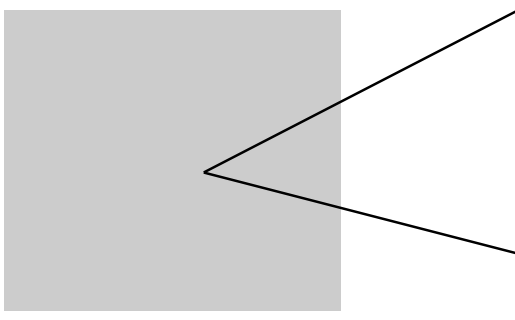

a)

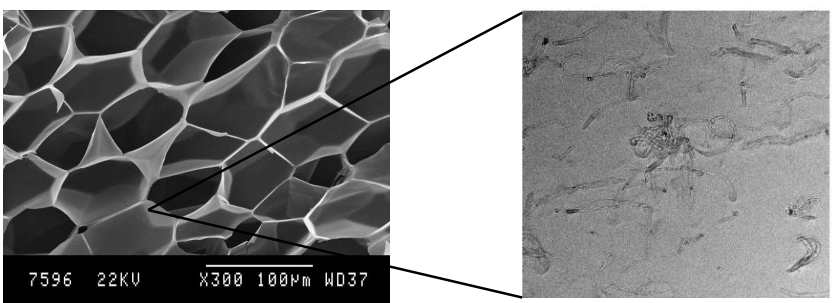

b) c)

Figure 1: The multiple scales involved in PP/CNTs nanocomposite foams: a) homogenized continuum at the macro-scale, b) foam micro-structure at the micro-scale, and c) $\mathrm{PP} / \mathrm{CNTs}$ nanocomposite made of cell walls at the nano-scale with the presence of CNTs and $\mathrm{PP}$ matrix (the matrix has amorphous and crystallized regions).

herein relies on the principle of scale separation, which is stated by

$$
L_{\text {macro }} \gg L_{\text {micro }} \gg L_{\text {nano }} .
$$

The left inequality of Eq. (1) leads to consider the structure made of foams as a homogeneous medium at the structural scale since the structural characteristic length is much larger than the cell size which varies from $20 \mu \mathrm{m}$ to $60 \mu \mathrm{m}$ as it will be shown in the experimental investigation later. The right inequality of Eq. (1) shows that the cell walls can be assumed as homogeneous at the micro-scale point-of-view. In general, a semi-crystalline polymer ( $\mathrm{PP}$ as a particular case) is observed as one made-up of a crystalline phase embedded into an amorphous phase under semi-crystalline spherical forms called spherulites. As discussed by Bao and Tjong (2008); Bédoui et al. (2006), each spherulite consists of many crystalline lamellae separately alternated by amorphous inter-layers. In the case of PP/CNTs nanocomposites, the optical micrographs show trans-crystalline inter-phases around the carbon fibers and the polypropylene spherulites away from them (Bikiaris, 2010). The trans-crystalline inter-phase is characterized by oriented crystalline lamellae surrounding the CNTs. As the lamellae thickness -which was measured to be of the order of several $\mathrm{nm}$ by Spieckermann et al. (2010) - and the CNTs diameter $-9.5 \mathrm{~nm}-$ are much smaller than the cell wall thickness -of the order of the $\mu \mathrm{m}$, this length-scale separation assumption across the cell walls holds.

From the principle of scale separation stated in Eq. (1), the modeling of structures made of nanocomposite foams follows two successive homogeniza- 
tion steps:

- Nano-micro homogenization: the properties of the foam cell walls are obtained through the homogenization of the PP/CNTs nanocomposite material. In particular, the effects of the nano-fillers and of the crystallinity of the polymer matrix on the properties of the obtained nanocomposite material are considered.

- Micro-macro homogenization: the foamed material properties at the structural level are predicted by homogenization of the micro-structure (i.e. the cells), using the homogenized cell wall properties obtained from the nano-micro homogenization step as input.

This constitutive behavior at the upper-scale (either at the micro-scale during the first homogenization step or at the macro-scale during the second homogenization step) can be evaluated either by using a phenomenological law or by developing a micro-mechanics model:

- Phenomenological laws are characterized by certain (semi-) analytic formulations whose parameters are identified from the numerical or experimental results by curve fitting and parameters identification Avalle et al., 2007). However this approach cannot predict the lower-scale structure evolution during the macroscopic loading and the required experiments are sometimes expensive and difficult to implement. Moreover, the sensitivity analyses required for material tailoring are not always possible.

- Nano/micro-mechanics models relate the equivalent properties of an heterogeneous material to their underlying lower-scale structure by using either analytical or numerical approaches. This approach allows material tailoring as the micro-structure is explicitly accounted for.

Both nano-mechanical models (Nikolov et al., 2002; van Dommelen et al., 2003; Seidel and Lagoudas, 2006; Bédoui et al., 2006; Parenteau et al., 2012) and phenomenological laws (Drozdov and Gupta, 2003; de Villoria and Miravete, 2007; Gan $\beta$ et al., 2008; Zrida et al., 2009) are suitable for the nanomicro homogenization step. Because the knowledge of the structural behavior at the nano-scale is still limited, phenomenological laws, validated via experimental results, are often preferred at this scale. Nevertheless, in order to keep the explicit dependency on the constituents properties in our numerical 
model, the mechanical parameters of the $\mathrm{PP} / \mathrm{CNT}$ s nanocomposite material are estimated by a new aggregate model, in which the degree of crystallinity and the volume percentage of nano-fillers are considered as the two input parameters. The accuracy of this theoretical model is assessed via conducted experimental measurements and from the literature. As the thickness of the cell walls is of the order of the $\mu \mathrm{m}$, it is difficult to directly measure their mechanical parameters, thus the model is validated with regards to raw $\mathrm{PP} / \mathrm{CNTs}$ nanocomposite samples of different crystallinity degrees. The cell walls properties can then be estimated via the degree of crystallinity obtained from DSC measurements on the foamed samples.

The micro-macro homogenization considers a structure made of foam as an homogeneous medium, whose constitutive model obeys to an equivalent stress-strain relationship obtained from the information of the underlying micro-structure. The most well-known micro-mechanics model is known as the Gibson and Ashby (1997) model, in which the constitutive behavior is divided into three stages: (i) linear elastic, (ii) plateau, and (iii) densification. As compared with other models, computational micro-mechanics is probably the most accurate approach to directly account for the micro-structure properties on the structural behavior (Daxner, 2010). In this approach, the finite element method (FEM) is used to discretize a representative volume element (RVE) extracted from the underlying micro-structure. Complex geometries and complex material behaviors of the micro-structure constituents can $a$ priori be considered with this approach. This approach is thus used to estimate the equivalent properties of the foamed material during the linear and plateau stages of the macro-scale response. To this end, a unit-cell is defined as a tetrakaidecahedron with variable cell wall thickness, face curvature, and cell wall waviness, to represent the foam cells and their imperfections.

The paper is organized as follows. Section 2 presents the experimental procedures and the related results. Section 3 details the development of a simple theoretical model to estimate the Young's modulus of PP/CNTs nanocomposites based on their CNTs contents and on the degree of crystallinity. The computational micro-mechanics model based on the tetrakaidecahedron unit cell is developed in Section 4. Finally, the obtained numerical results at the macro-scale are compared with experimental results in Section 5 showing that the proposed unit cell computational micro-mechanics approach can be used to model PP/CNTs nanocomposite foams. 


\section{Experimental investigation}

In this section, the experimental process followed to obtain the different $\mathrm{PP} / \mathrm{CNTs}$ nanocomposite raw and foamed samples is summarized. The measurement of the PP/CNTs nanocomposite Young's modulus could not be performed directly on the cell walls of the foamed samples as their thickness is of the order of the $\mu \mathrm{m}$. Instead three-point bending tests of raw PP/CNTs nanocomposite samples with different crystallinity degrees were conducted. As a result, the cell walls properties can be estimated via their degree of crystallinity obtained from DSC measurements on the foamed samples. Finally compressive tests on the foamed samples are detailed. These tests will be used to validate the multi-scale models presented in this paper.

\subsection{Material preparation}

Polypropylene $(\mathrm{PP})-$ Eltex $^{\mathrm{R}} \mathrm{P}$ KS407 is a random copolymer with a high ethylene content. It has a density of $895 \mathrm{~kg} / \mathrm{m}^{3}$, and its melting point is $134{ }^{\circ} \mathrm{C}$.

Carbon nanotubes (CNTs)- Nanocyl ${ }^{\mathrm{TM}}$ NC 7000 Thin Multiwall Carbon Nanotubes (MWCNTs) - are produced by a Chemical Vapor Deposition (CVD) process. The produced CNTs have a surface area of $250-300 \mathrm{~m}^{2} / \mathrm{g}$ and a carbon purity of $90 \%$. The average diameter and length 11 of these CNTs are $9.5 \mathrm{~nm}$ and 1.5 microns, respectively.

The foamed materials were produced using super-critical $\mathrm{CO}_{2}\left(\mathrm{ScCO}_{2}\right)$ - Carbon dioxide with $99.8 \%$ purity was purchased from Sigma ${ }^{\circledR}, 501298$ cylinder 48L. During the foaming process, $\mathrm{CO}_{2}$ was introduced into the stainless steel vessel using a syringe pump, TELEDYNE ISCO Model 260D. The cylinder volume of the pump is $266 \mathrm{ml}$, the pressure ranges from 0 to 7500 psi, and the flow ranges from 0.001 to $70 \mathrm{ml} /$ minute.

The parameters of the preparation procedures are summarized in Fig. 2.

\subsubsection{Preparation of $P P / C N T$ s nanocomposite material}

Melt mixing is used to prepare the $\mathrm{PP} / \mathrm{CNT}$ s nanocomposite material because this method leads to a good dispersion of the CNTs in the PP matrix. First, the PP matrix and two different weight percentages of CNTs

\footnotetext{
${ }^{1}$ Practically their length could be lower due to cutting during the melt-mixing process, but this does not affect the model.
} 


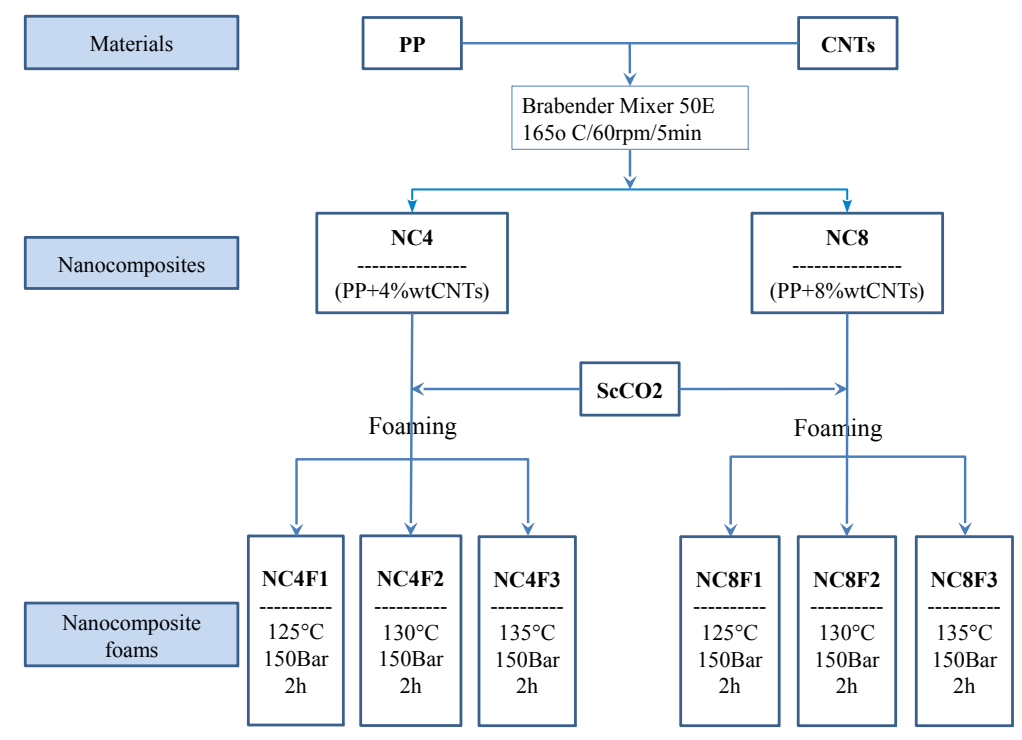

Figure 2: $\mathrm{PP} / \mathrm{CNT}$ s nanocomposite material and foamed samples preparation.

Table 1: Environmental conditions of the NC4 samples in order to modify their crystallinity.

\begin{tabular}{lc}
\hline & Description \\
\hline NC4C1 & After nanocomposite preparation \\
\hline NC4C2 & In the stainless steel vessel: $125{ }^{\circ} \mathrm{C}, 150$ bar, $4 \mathrm{~h}$ \\
\hline NC4C3 & In the stainless steel vessel: $125{ }^{\circ} \mathrm{C}, 150$ bar, $8 \mathrm{~h}$ \\
\hline
\end{tabular}

(4 wt\% and $8 \mathrm{wt} \%$ ) were mixed in a Brabender Mixer -50E (Model 835205002) Roller Slade twin-screws- at $165^{\circ} \mathrm{C}$ with 60 rotations per minute during 5 minutes. The $4 \mathrm{wt} \% \mathrm{PP} / \mathrm{CNTs}$ and $8 \mathrm{wt} \% \mathrm{PP} / \mathrm{CNT}$ s nanocomposites are denoted as $\mathrm{NC} 4$ and $\mathrm{NC} 8$ as shown in Fig. 2.

The samples for the three-point bending tests were molded into $4.5 \times$ $12 \times 60$ mm-rectangular prisms by hot pressing -Fortune SRA100- of the nanocomposite material at $180{ }^{\circ} \mathrm{C}$ for 5 minutes. Since the foaming process can affect the crystallinity of the $\mathrm{PP} / \mathrm{CNT}$ s nanocomposite materials, several NC4 nanocomposite samples were left, after preparation, into the stainless vessel under the same conditions as for the foaming process, i.e. saturated in $\mathrm{ScCO}_{2}$, at the same temperature and pressure, but without performing the 
foaming to observe the change of crystallinity. These conditions are detailed in Tab. 1. After specific soaking times $(0 \mathrm{~h}, 4 \mathrm{~h}$, and $8 \mathrm{~h})$, the vessels is cooled down in an ice bath and the pressurized $\mathrm{CO}_{2}$ is slowly released so that the foams are not created. Finally, these samples are held under normal ambient conditions during a few days to release the absorbed $\mathrm{CO}_{2}$ and to stabilize the composite structure. The DSC tests were performed on these final samples. Note that since the crystallinity degree evolves with time, the DSC measurements and the mechanics tests were performed the same week.

\subsubsection{Preparation of $\mathrm{PP} / \mathrm{CNTs}$ nanocomposite foams using $\mathrm{ScCO}_{2}$}

The un-foamed material (cut from large NC4 and NC8 samples) were used as starting materials for the foaming process. The foamed samples were prepared using a batch process as previously performed by Tran et al. (2013).

First, different NC4 and NC8 samples were separately placed into the stainless steel vessel. Subsequently, $\mathrm{CO}_{2}$ was pressurized into the vessel by using syringe with high pressure-liquid pump to get the super-critical state. When the required pressure (150 bar) was achieved, the system was kept at constant pressure and different soaking temperatures during 2 hours to ensure a sufficient saturation amount of $\mathrm{CO}_{2}$ and to reach its thermodynamic solubility. After soaking, a rapid depressurization was applied. Finally, the vessel was then immersed immediately into a water/ice bath in order to stabilize the foams.

The sample names (NC4F1, NC4F2, NC4F3, NC8F1, NC8F2 and NC8F3) and their respective foaming conditions are summarized in Fig. 2. The DSC tests were performed on the final samples. As for the unfoamed samples, since the crystallinity degree evolves with time, the DSC measurements and the mechanics tests were performed the same week.

\subsection{Characterizations}

\subsubsection{Micro-structure of PP/CNTs nanocomposite foams}

In order to obtain details on the micro-structure of the processed nanocomposite foams, six different -NC4F1, NC4F2, NC4F3, NC8F1, NC8F2 and NC8F3- representative samples were immersed into nitrogen liquid for 30 minutes and then quickly fractured. The foam structure was observed by scanning electron microscopy (SEM) using a JEOL JSM 840-A microscope after metallization with $\mathrm{Pt}(30 \mathrm{~nm})$. Typical micro-graphical images are shown in Fig. 3. The only changes in the preparation of these six samples 


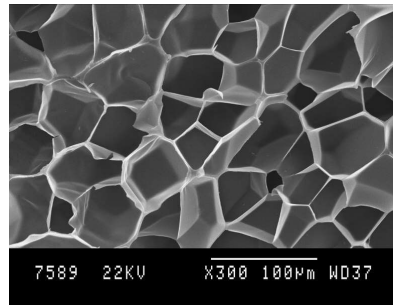

(a) $\mathrm{NC} 4 \mathrm{~F} 1$

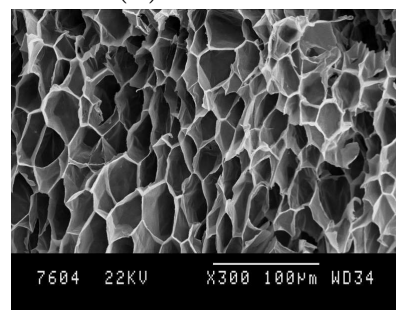

(d) $\mathrm{NC} 8 \mathrm{~F} 1$

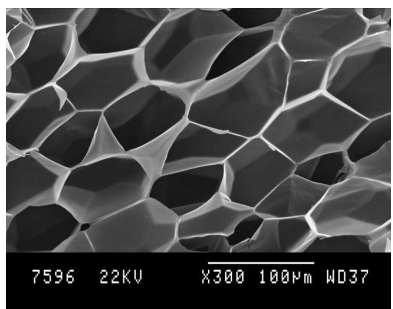

(b) $\mathrm{NC} 4 \mathrm{~F} 2$

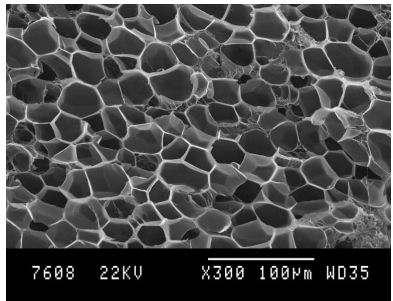

(e) NC8F2

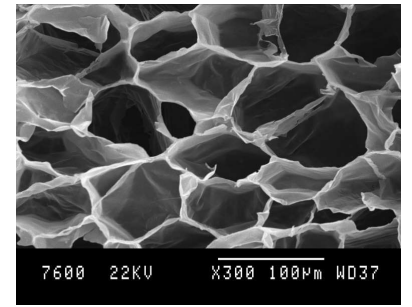

(c) NC4F3

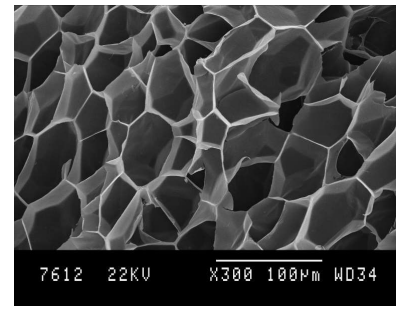

(f) NC8F3

Figure 3: Typical micro-graphical images of $4 \% \mathrm{wt} \mathrm{PP} / \mathrm{CNTs}$ and $8 \% \mathrm{wt} \mathrm{PP} / \mathrm{CNTs}$ nanocomposite foams (refer to Fig. 2 for samples code).

are the soaking temperature and the CNTs content, therefore the differences in the micro-structure depend on these two factors. The cell disorder is qualitatively seen to be increased with the soaking temperature, see Fig. 3. By using the statistical methods reported by Tran et al. (2013), several micro-structure characteristics can be estimated, including the relative density (which is defined by the ratio $\rho / \rho_{s}$ where $\rho$ is the density of the foam and where $\rho_{s}$ is the density of the material from which this foam is made). The obtained results are depicted in Fig. 4 as functions of the soaking temperature and of the CNTs contents.

Fig. 4a shows that the increase of the soaking temperature leads to larger pore sizes and that, under the same foaming condition, a smaller CNTs content leads to higher pore sizes. Fig. 4b plots the evolution of the cell density with respect to the soaking temperature. Adversely to the pore size, the cell density decreases with the soaking temperature, which agrees with the theory of nucleation as pointed out by Tran et al. (2013). Since the nanofillers are acting as nucleating agents, their increase results in a higher cell density and leads to smaller pore sizes. The $8 \% \mathrm{wt}$ CNTs foamed samples exhibit a larger trend compared to the $4 \%$ wt CNTs ones. Fig. 45 shows the dependency of the volume expansion ratio on the soaking temperature. 


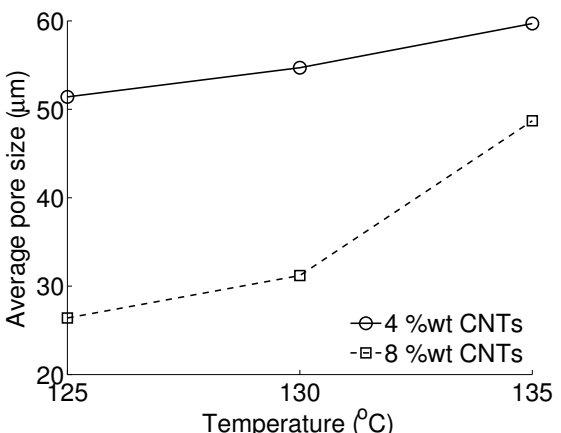

(a)

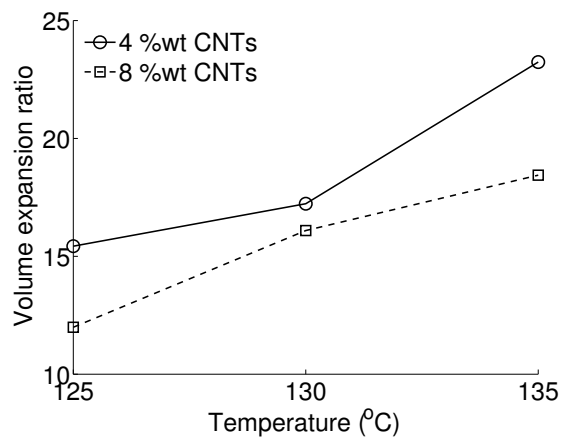

(c)

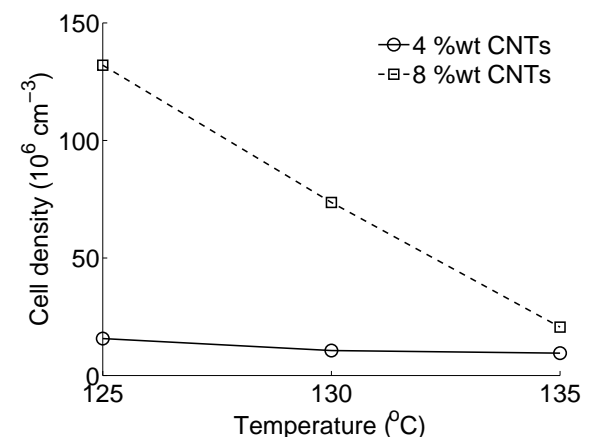

(b)

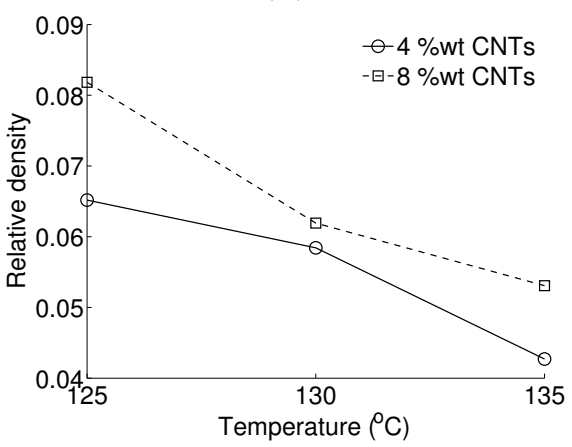

(d)

Figure 4: Dependence of foam characteristics on the soaking temperature: (a) average pore size, (b) cell density, (c) expansion volume ratio, and (d) relative density.

The volume expansion ratio increases with the temperature with a similar trend for the two CNTs contents. Adversely to the volume expansion ratio, the relative density decreases with the soaking temperature for both CNTs contents, as illustrated by Fig. $4 \mathrm{~d}$.

Many imperfections are found in the material micro-structure (see Fig. 3) such as variable cell sizes and shapes, cell wall fracture, cell wall curvature, missing cell walls, cell wall corrugation, etc. These imperfections do not result from the film preparation. Higher CNTs contents (from $4 \%$ to $8 \%$ ) and higher soaking temperatures (from $125^{\circ} \mathrm{C}$ to $135^{\circ} \mathrm{C}$ ) result in an increase of those imperfections. Moreover, during the preparation of nanocomposites, a higher content of CNTs can lead to a poorer dispersion of the CNTs in the PP matrix with the presence of bigger agglomerates. When foaming these nanocomposites with big agglomerates under high pressure conditions, the fracture of the cell walls can more easily occur due to high stress concentra- 
tion. Finally, because the modulus of PP matrix reduces when increasing the temperature, a higher soaking temperature increases the imperfections. As those imperfections lead to the reduction of the stiffness of the foamed structures, they will be taken into account during the modeling stage by considering (i) closed cells with thinner cell walls up to reaching open cells, (ii) cell wall curvatures, and (iii) face waviness.

\subsubsection{Differential Scanning Calorimetry}

In semi-crystalline polymers such as PP, the volume percentage of the crystalline phase (so-called degree of crystallinity) directly affects their physical properties, such as the mechanical properties, storage modulus, permeability, melting point, etc. The nanocomposite base material and the foaming preparation conditions - the temperature changes, foaming agents, and nanofillers properties- can affect the crystallinity of the PP matrix as shown by Takada et al. (2001); Leelapornpisit et al. (2005). The change of the crystallinity degree leads to different mechanical behaviors of the PP phase in neat $\mathrm{PP}$, in nanocomposites, and in the cell walls of nanocomposite foams. Thus a direct measurement of the degree of crystallinity is necessary and provides fundamental results to predict other useful properties.

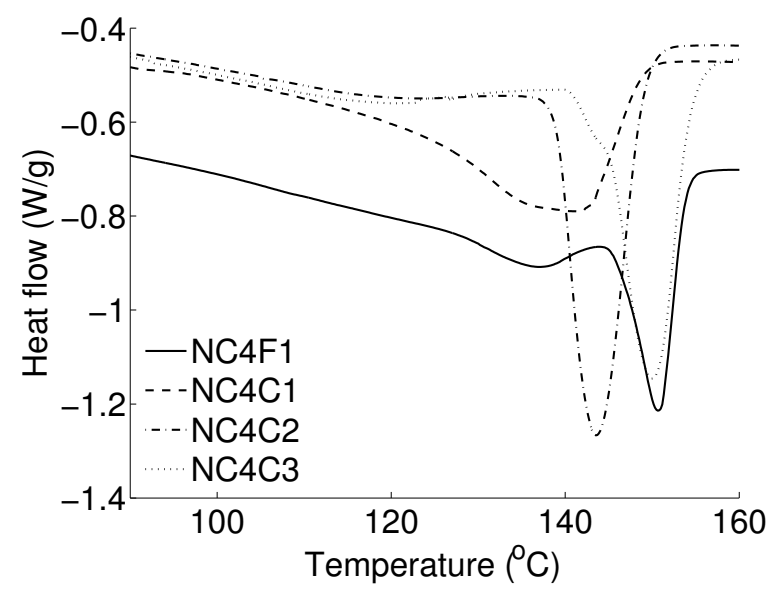

Figure 5: DSC thermograms for the first heating cycle for the different NC4 samples and for the NC4F1 sample.

Differential Scanning Calorimetry (DSC) techniques are generally used to characterize the polymer degree or crystallinity and are herein performed on the (un-foamed) NC4C1, NC4C2, NC4C3 samples, see Tab. 1, and on the 
Table 2: DSC crystallinity characteristics of $4 \%$ wt PP/CNTs nanocomposites (refer to Tab. 1 for details of the soaking conditions) and of foamed samples (refer to Fig. 2 and Tab. 1 for samples code).

\begin{tabular}{lcc} 
Sample code & $\Delta \mathrm{H}(\mathrm{J} / \mathrm{g})$ & $\mathrm{X}_{c}(\%)$ \\
\hline NC4C1 & 35.4 & 17.6 \\
\hline NC4C2 & 29.9 & 14.9 \\
\hline NC4C3 & 26.9 & 13.4 \\
\hline NC4F1 & 16.1 & 8.0 \\
\hline NC4F2 & 18.5 & 9.2 \\
\hline NC4F3 & 14.4 & 7.2 \\
\hline NC8F1 & 16.4 & 8.5 \\
\hline NC8F2 & 17.2 & 9.0 \\
\hline NC8F3 & 14.0 & 7.4 \\
\hline
\end{tabular}

foamed samples. Figure 5 illustrates the DSC curves of the NC4 nanocomposite samples exposed under the different conditions reported in Tab. 1, and of the NC4F1 foamed sample. The degree of crystallinity $\left(X_{c}\right)$ is tabulated in Tab. 2 for the NC4 nanocomposite samples with different soaking conditions and for the different NC4 and NC8 foamed samples. The volume percentage of the crystalline phase in the PP matrix (so-called the degree of crystallinity) is quantified by the expression proposed by Soitong and Pumchusak (2011)

$$
\mathrm{X}_{c}(\%)=\frac{\Delta \mathrm{H}}{(1-w) \Delta \mathrm{H}_{0}} 100
$$

where $w$ is the weight percentage of nanocomposite fillers, $\Delta H$ is the crystallization enthalpy estimated from the DSC curves in Fig. 5, and where $\Delta \mathrm{H}_{0}=209 \mathrm{~J} / \mathrm{g}$ for $100 \%$ crystalline polypropylene.

A notable change of the crystallinity is observed for the different samples, see Tab. 2. The crystallization enthalpy $(\Delta \mathrm{H})$ decreases with the soaking time of the un-foamed samples and is larger than for their corresponding foamed samples. Indeed, the foamed materials exhibit a lower crystallinity $\left(X_{c}=8.0 \%\right.$ for the NC4F1 sample, $X_{c}=9.2 \%$ for the NC4F2 sample, and $X_{c}=7.2 \%$ for the NC4F3 sample) compared to the initial respective unfoamed material $\left(X_{c}=17.6 \%\right.$ ), which means that their mechanical properties will differ. If for the un-foamed material the degree of crystallinity decreases with the soaking time, it is however not sensitive to the CNTs content. 
Table 3: Elastic flexural modulus of PP/CNTs nanocomposites (refer to Tab. 1 for samples code).

\begin{tabular}{lccc}
\hline & neat PP $(\mathrm{MPa})$ & $\mathrm{NC} 4(\mathrm{MPa})$ & $\mathrm{NC} 8(\mathrm{MPa})$ \\
\hline $\mathrm{NC} 4 \mathrm{C} 1$ & $622.6 \pm 4.2$ & $785.0 \pm 10.0$ & $904.4 \pm 27.5$ \\
\hline NC4C2 & 503.7 & $503.0 \pm 13.1$ \\
\hline NC4C3 & \multicolumn{4}{c}{480.6} \\
\hline
\end{tabular}

\subsection{Three-point bending tests of PP/CNTs nanocomposite materials}

The three-point bending tests were performed to measure the elastic flexural modulus of the $\mathrm{PP} / \mathrm{CNTs}$ nanocomposite materials using an Instron 5500 machine at room temperature. A small displacement rate $(0.5$ $\mathrm{mm} /$ minute) was used so that the test condition can be considered as quasistatic. Different samples of neat PP, NC4, and NC8 were used to obtain average results. The elastic flexural modulus are obtained by linearizing the force/displacement reaction curves on the initial elastic region and are reported in Tab. 3 .

On the one hand, the addition of CNTs in the PP matrix results in an increase of the average flexural modulus of the nanocomposites (from 622.6 $\mathrm{MPa}$ for neat PP samples to $785 \mathrm{MPa}$ for NC4 samples and to 904.4 for $\mathrm{NC} 8$ samples, see Tab. 3). On the other hand, the flexural modulus is reduced when increasing the soaking time (from 622.4 MPa to 503.7 MPa for neat PP samples and from $785 \mathrm{MPa}$ to $480.6 \mathrm{MPa}$ for NC4 samples), which is consistent with the reduction of the degree of crystallinity reported in Tab. 2.

\subsection{Uniaxial compression tests of PP/CNTs nanocomposite foams}

The uniaxial compression tests are performed to characterize the compressive behavior of the $\mathrm{PP} / \mathrm{CNT}$ s nanocomposite foams. They are carried out on $5 \mathrm{~mm}$-cubic foamed samples, which are cut from different locations of larger foam blocks of the NC4F1, NC4F2, NC4F3, NC8F1, NC8F2 and NC8F3 samples. The uniaxial compression tests were performed using an Instron 5500 machine at room temperature under quasi-static conditions (displacement rate of $0.5 \mathrm{~mm} /$ minute). All foamed samples were compressed until reaching a deformation of $80 \%$. The compression tests were performed with force-free lateral surfaces and the friction between the samples and the machine platens was not controlled. However, during the compression tests, the PP foamed specimens collapse with negligible Poisson ratio's effect as 
reported by Zhang et al. (1998). As a result, although the local state can be different (Youssef et al., 2005, Ryu et al., 2005), the effect of the friction conditions on the overall stress-strain response can be neglected compared to the response discrepency between the different specimen responses, see the results here below.

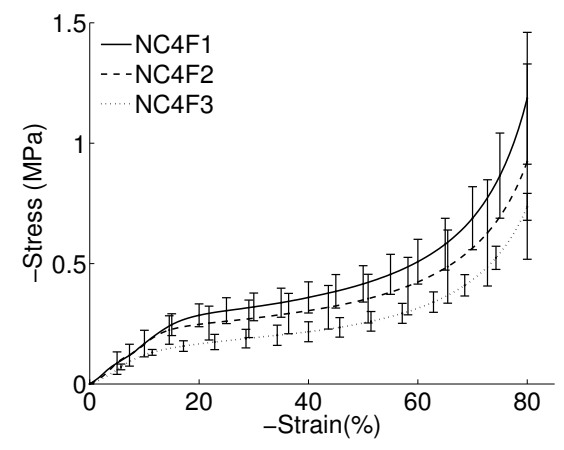

(a) $4 \%$ wt CNTs

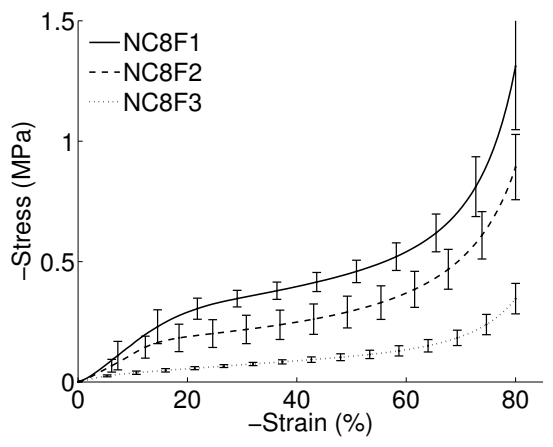

(b) $8 \%$ wt CNTs

Figure 6: Uniaxial compressive stress-strain curves of the PP/CNTs nanocomposite foams: (a) $4 \%$ wt CNTs samples and (b) $8 \%$ wt CNTs samples.

The resulting uniaxial compressive curves of the nanocomposite foams are presented in Fig. 6. Three different stages can be distinguished: (i) the linear elastic region at low compressive strain, (ii) the stress plateau region, and (iii) the densification region at high compressive strain. The mechanical properties of the foamed samples depend on the properties of the base material, but also on its relative density and on its micro-structure (cell size, cell shape, cell distribution, etc). Therefore, the differences into the micro-structural characteristics depicted in Fig. 4 are reflected in the compression curves reported in Fig. 6.

The homogenized (so-called macroscopic) elastic modulus, or Young's modulus, is estimated from the initial linear-elastic region of the compressive curves. The collapse strength is defined by the intersection between the linearized curve of the elastic region and the linearized curve of the stress plateau region. The results of the Young's modulus and of the collapse strength of the different nanocomposite foams are reported in Fig. 7 in terms of their relative density, with their error bars expressing their standard deviation. Clearly, for each CNTs contents (4\%wt and $8 \%$ wt), the Young's modulus and collapse strength increase when increasing the relative density. Moreover, it can be seen that the nanocomposite foams with higher CNTs 


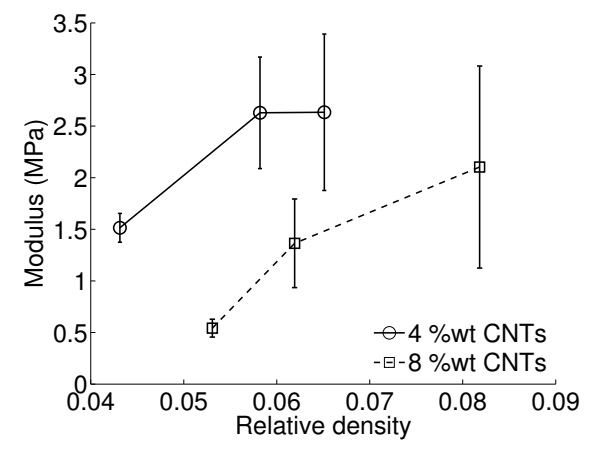

(a)

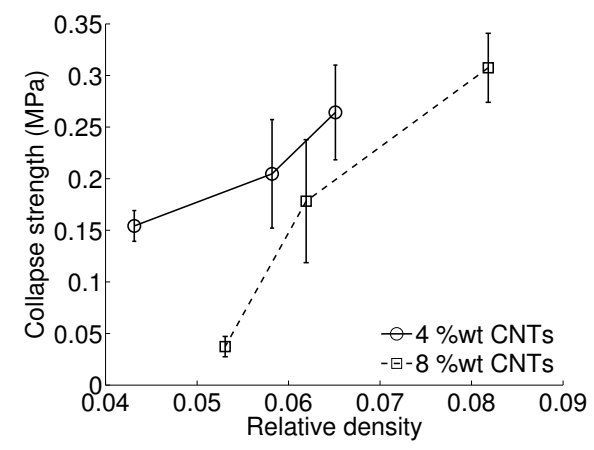

(b)

Figure 7: Elastic modulus (a) and collapse strength (b) of the PP/CNTs nanocomposite foams in terms of their relative density.

content have lower Young's modulus and lower collapse strength at the same relative density. These differences can be attributed from different levels of micro-structure imperfections as shown in Fig. 3 and from the degree of crystallinity which is inevitably modified during the foaming process as discussed in Section 2.2.2. Due to the presence of a higher CNTs content, a poor dispersion of the CNTs in the PP matrix during the preparation process results in the presence of bigger agglomerates. The fracture of the cell walls can thus more easily occur due to high stress concentration during the foaming process under high pressure conditions. Moreover, because the modulus of the PP matrix reduces when increasing the temperature, a higher soaking temperature increases the imperfections. These increasing imperfections lead to the reduction of the stiffness of the foamed structures. In spite of this, a higher CNTs content can improve the electrical and electromagnetic properties for specific applications as discussed by Thomassin et al. (2010); Tran et al. (2013).

\section{Nano-micro homogenization: theoretical evaluation of PP/CNTs nanocomposite materials Young's modulus}

In order to estimate the homogenized properties of the nanocomposite foams using a computational micro-mechanics model, the material behavior of the cell walls has first to be identified. As demonstrated in the experimental part, the nanocomposite and foam preparations affect the properties of the PP matrix so that the properties of the foam cell walls do not correspond 
exactly to the properties of the original nanocomposites. In addition, the cell walls consist of thin structures with small dimensions (magnitude order of the $\mu \mathrm{m}$ ) so it is difficult to measure exactly their material parameters. An effective theoretical model to estimate the Young's modulus of cell walls based on their degree of crystallinity and on the CNTs content is thus developed.

Semi-crystalline polymers (PP as a particular case) are observed as one made- up of a crystalline phase embedded into an amorphous phase under the semi-crystalline spherical shapes called spherulites. Each spherulite consists of many crystalline lamellae separately alternated by amorphous inter-layers as shown by Bao and Tjong (2008); Bédoui et al. (2006). In case of PP/CNTs nanocomposites, the optical micrographs show trans-crystalline inter-phases around the carbon fibers and the polypropylene spherulites away from the carbon fibers Bikiaris (2010). The trans-crystalline inter-phase is characterized by oriented crystalline lamellae surrounding the carbon fibers. Therefore these nanocomposites exhibit complex mechanical behaviors since the crystalline phase, the amorphous phase, and the carbon nanotubes are combined together in a complex micro-structure. Using the assumption that there is no molecular modification of the polymer during the PP/CNTs nanocomposite preparation, the crystallinity of the $\mathrm{PP}$ matrix is the key parameter that has an effect on the mechanical properties of the composite material. Because of the foaming condition process, the degree of crystallinity of the PP matrix is reduced as demonstrated in Tab. 2, leading to the reduction of the cell walls Young's modulus.

Several micro-mechanics models have been proposed in the literature for semi-crystalline polymers, (Nikolov et al., 2002, van Dommelen et al., 2003; Seidel and Lagoudas, 2006; Bédoui et al., 2006) as a non exhaustive list. In particular Nikolov et al. (2002) have considered a crystalline visco-plasticity model for the lamellae and a visco-elastic model for the the amorphous phase. The homogenized behavior of a stack of parallel crystalline/amorphous layers is obtained by assuming a uniform stress in the stacked material. The Sachs homogenization model (uniform stress) is then used to extract the meso-scale homogenized behavior of the material having two-phase layered materials of different orientations. van Dommelen et al. (2003) have considered (crystalline) visco-plasticity for both the lamellae and the amorphous phases assembled in a two-phase layered inclusion. They have also analyzed different homogenization models, such as Taylor (uniform strain), Sachs (uniform stress), and hybrid models, to predict the meso-scale behavior of this two-phase layered material. The hybrid model considering a uniform stress 
in the direction perpendicular to the amorphous-crystalline interface and a uniform strain in the directions parallel to this interface has been shown to predict the more physical results. The different orientations of the lamellae are accounted for by considering an aggregate of two-phase layered inclusions having different orientations, the hybrid scheme being applied in the projected referential of each inclusion interface. Elastic properties of semicrystalline materials have been studied by Bédoui et al. (2006). On the one hand they have considered the crystalline phase as inclusions and performed an homogenization step using a self-consistent scheme, possibly enhanced by a dilute scheme in which the crystalline fraction is increased step-by-step. On the other hand, the two-phase layered-inclusions, whose properties are obtained using the hybrid model, are homogenized to account for the different lamellae directions by using either the aggregate model proposed by van Dommelen et al. (2003) or a self-consistent scheme. It was shown that for PP materials, as the lamellae have a high aspect ratio, both the model of crystalline inclusions combined to a dilute self-consistent homogenization (referred to as the Matrix-Inclusion Dilute Scheme Method) and the model of two-phase layered inclusions combined to an aggregate model (referred to as the U-inclusion approach) lead to acceptable predictions of the elastic behavior as compared to experiments. The advantage of the latter being the absence of a shape parameter - difficult to evaluate in most cases- requirement (Bédoui et al., 2006). However, these models do not account for the CNTs reinforcement.

During the compressive loading of the foamed materials, since the samples have a crystallinity degree lower than $10 \%$, see Tab. 2, the elastic collapse mode has to be captured with accuracy to model the plateau in the stress-strain curve (Gibson and Ashby, 1997, e.g.). Moreover as it will be shown in Section 5.2, the local strain rate in the foamed material walls is of the same order of magnitude as the global strain rate, motivating the development of an elastic-model without strain-rate effects. Thus the elastic behavior of the PP/CNTs nanocomposite material has to be correctly predicted. As the shape parameter of the PP lamellae is not well known, a new aggregate model of two-phase layered inclusions of PP/CNTs nanocomposites is constructed in Section 3.1. Each layered inclusion contains a crystalline part and a CNTs/amorphous part, in which the CNTs is assumed to be randomly dispersed in the amorphous matrix. The Young's modulus of the CNTs/amorphous part is estimated using the Halpin-Tsai model as described by Affdl and Kardos (1976), which is based on the self-consistent 
method in conjunction with the Tsai and Pagano (1968) model. The homogenous Young's modulus of randomly dispersed inclusions following the Tsai-Pagano model, which was first developed to predict the elastic modulus of composites containing short fibers randomly oriented in a plane, is predicted by

$$
E_{\text {random }}=\frac{3}{8} E_{L}+\frac{5}{8} E_{T},
$$

where $E_{L}$ and $E_{T}$ are respectively the longitudinal and transverse moduli of an inclusion. ${ }^{2}$ Once the Young's modulus of the CNTs/amorphous phase is estimated using Eq. (3), the homogenized behavior of the crystalline PP / reinforced amorphous PP layered inclusions can then be obtained using the hybrid model, leading to an expression of the Young's modulus which depends on the degree of crystallinity and on the content of carbon nanotubes. Since this meso-scale homogenized behavior is orthotropic, the isotropic behavior is obtained by considering a random dispersion of layered inclusions using the Tsai-Pagano model (3) a second time. The analytical results are validated from the experiments performed in Section 2, but also from the literature. Therefore, using the degrees of crystallinity of the foamed samples reported in Tab. 2, the Young's modulus of the cell walls can be estimated for the different foamed materials.

\subsection{The aggregate model}

The schematic description of the aggregate model is reported in Fig. 8 , The contributions of the separate composite components (CNTs, crystalline phase, amorphous phase) are known from the volume percentage of CNTs and from the degree of crystallinity $\left(X_{c}\right)$. The two-phase layered element is characterized by the CNTs/amorphous part (denoted by $a$ ) and by the crystalline part (denoted by $c$ ). The behavior of the CNTs/amorphous part is isotropic since the CNTs are randomly distributed into an isotropic amorphous PP matrix. Its Young's modulus is estimated following the work of

\footnotetext{
${ }^{2}$ The formulation (3) is based on the averaging technique over the fiber orientation in a plane under the constant strain assumption. Although this assumption is more suitable for laminates and sheet materials, this simple model can be used to estimate the mechanical behavior of nano-composites, see (Coleman et al., 2006, de Villoria and Miravete, 2007; Gan $\beta$ et al. 2008: Pedrazzoli and Pegoretti $\mid 2014)$ e.g. and of short fiber composites, see (Lavengood and Goettler, 1971, López et al., 2012, Serrano et al., 2014, Mortazavian and Fatemi, 2015) e.g without significant computational effort.
} 


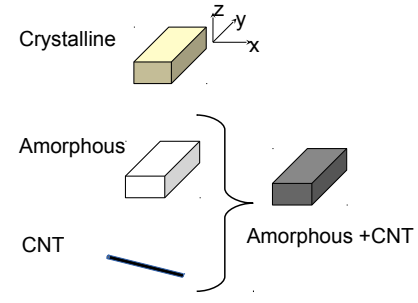

(a) Constituents

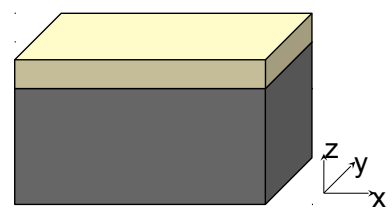

(b) Layered element

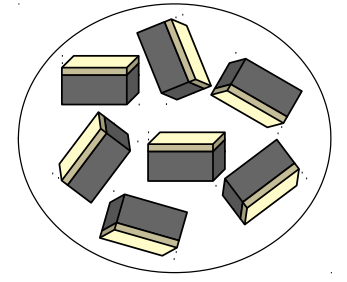

(c) Random distribution

Figure 8: Aggregate model for CNTs/semi-crystalline polymer nanocomposites: (a) different constituents of the composites, (b) representative layered element with the CNTs/amorphous part and the crystalline part, and (c) random distribution of aggregate elements. The lamellae growth, width, and chains follow respectively $\mathrm{x}-, \mathrm{y}_{-}$, and $\mathrm{z}-$ directions.

de Villoria and Miravete (2007); Gan $\beta$ et al. (2008) who have extended the relation (3) as

$$
\begin{gathered}
E_{a}=\left[\frac{3}{8}\left(\frac{1+2 \alpha \eta_{L} V_{\mathrm{CNT}}^{a}}{1-\eta_{L} V_{\mathrm{CNT}}^{a}}\right)+\frac{5}{8}\left(\frac{1+2 \eta_{T} V_{\mathrm{CNT}}^{a}}{1-\eta_{T} V_{\mathrm{CNT}}^{a}}\right)\right] E_{m} \\
\text { with } \alpha=\frac{l}{d}, \eta_{L}=\frac{\frac{\mathrm{E}_{\mathrm{CNT}}}{\mathrm{E}_{m}}-1}{\frac{\mathrm{E}_{\mathrm{CNT}}}{\mathrm{E}_{m}}+2 \alpha}, \text { and } \eta_{T}=\frac{\frac{\mathrm{E}_{\mathrm{CNT}}}{\mathrm{E}_{m}}-1}{\frac{\mathrm{E}_{\mathrm{CNT}}}{\mathrm{E}_{m}}+2},
\end{gathered}
$$

where $E_{m}, E_{\mathrm{CNT}}$, and $E_{a}$ are the Young's moduli of the amorphous PP, of the carbon nanotubes, and of the CNTs/amorphous PP, respectively, $l$ and $d$ are the average length and diameter of the carbon nanotubes, and where $V_{\mathrm{CNT}}^{a}$ is the volume percentage of CNTs in the amorphous PP matrix -which is different from the volume percentage of the CNTs in the nanocomposite material.

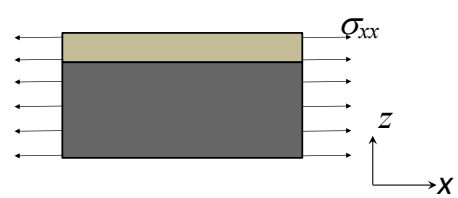

(a) $\mathrm{x}$-direction

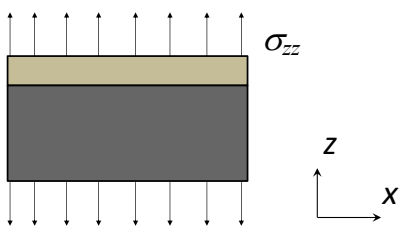

(b) z-direction

Figure 9: Uniaxial loading in different directions on a layered element. 
The behavior of the crystalline part is orthotropic following the $x^{-}, y^{-}$, and $z$-directions, as presented in Fig. 8. The corresponding Young's moduli are $E_{c x x}, E_{c y y}$, and $E_{c z z}$, in which the $x-, y-$, and $z$-directions correspond to the lamellae growth, width and chains directions respectively. As the lamellae are much longer along the $x$-direction, about 100 to 1000 times, in the representative two-phase layered element illustrated in Fig. 8b, the deformation compatibility, under loading along the $x$-direction, see Fig. 9 a, is guaranteed by adding the constituent stress; leading to

$$
\sigma_{x x} A=\sigma_{c x x} f_{c} A+\sigma_{a x x}\left(1-f_{c}\right) A
$$

where $A$ is the cross section area, $\sigma_{x x}, \sigma_{c x x}$, and $\sigma_{a x x}$ are the equivalent stresses of the aggregate element, in the crystalline part, and in the CNTs/amorphous part, respectively, and where $f_{c}$ is the volume percentage of the crystalline phase in the nanocomposite material. This volume percentage is given by

$$
f_{c}=\left(1-V_{\mathrm{CNT}}\right) X_{c}
$$

where $X_{c}$ is degree of crystallinity and where $V_{\mathrm{CNT}}$ is the volume percentage of CNTs in the nanocomposite material. Since

$$
\sigma_{x x}=E_{x x} \varepsilon_{x x}, \quad \sigma_{c x x}=E_{c x x} \varepsilon_{x x}, \text { and } \quad \sigma_{a x x}=E_{a} \varepsilon_{x x}
$$

where $\varepsilon_{x x}$ is the strain along the $x$-direction, the equivalent Young's modulus along the $x$-direction of the representative aggregate element is given by

$$
E_{x x}=f_{c} E_{c x x}+\left(1-f_{c}\right) E_{a} .
$$

The same process is applied in the $y-z$ plane leading to

$$
E_{y y}=f_{c} E_{c y y}+\left(1-f_{c}\right) E_{a}
$$

where $E_{y y}$ is the equivalent Young's modulus of the representative aggregate element along the $y$-direction.

When applying a uniaxial stress in the $z$-direction, as depicted in Fig. 9b, the stress-compatibility is guaranteed by adding the constituent strains as

$$
\varepsilon_{z z}=f_{c} \varepsilon_{c z z}+\left(1-f_{c}\right) \varepsilon_{a z z}
$$


where $\varepsilon_{x x}, \varepsilon_{c x x}$, and $\varepsilon_{a x x}$ are the equivalent strain on the aggregate element, the strain in the crystalline phase, and the strain in the CNTs/amorphous phase, respectively. Since

$$
\varepsilon_{z z}=\frac{\sigma_{z z}}{E_{z z}}, \quad \varepsilon_{c z z}=\frac{\sigma_{z z}}{E_{c z z}} \text { and } \quad \varepsilon_{a z z}=\frac{\sigma_{z z}}{E_{a}}
$$

the equivalent Young's modulus along the $z$-direction of the representative aggregate element is given by

$$
E_{z z}=\left(\frac{f_{c}}{E_{c z z}}+\frac{1-f_{c}}{E_{a}}\right)^{-1} .
$$

Finally, following the work of Lavengood and Goettler (1971); López et al. (2012); Serrano et al. (2014); Mortazavian and Fatemi (2015), the isotropic behavior, resulting from the random distribution of the orthotropic layered material, can be approximated by Eq. (3). In our case, as $E_{x x} \neq E_{y y}$, the following isotropic modulus is proposed:

$$
E_{s}=\frac{3}{8} \frac{\left(E_{x x}+E_{y y}\right)}{2}+\frac{5}{8} E_{z z}
$$

where $E_{x x}, E_{y y}$, and $E_{z z}$ are given by Eqs. (8), (9), and (12), respectively.

The theoretical model given by Eq. (13) shows the dependency of the $\mathrm{PP} / \mathrm{CNTs}$ nanocomposite properties on both the CNTs percentage and on the degree of crystallinity of the material.

\subsection{Comparison of the theoretical model predictions with literature data}

The input data considered to model the nanocomposite materials are the following ones:

- The average diameter and length of the CNTs are $9.5 \mathrm{~nm}$ and $1.5 \mathrm{mi}$ crons ${ }^{3}$, respectively. The theoretical value $E_{\mathrm{CNT}} \approx 1 \mathrm{TPa}$ is considered for the CNTs;

- The Young's modulus of the amorphous phase is estimated to be $E_{m}=$ $0.9 \mathrm{MPa}$, following the work of Bédoui et al. (2006), and;

\footnotetext{
${ }^{3}$ Practically their length could be lower due to cutting during the melt-mixing process, but this does not affect the model.
} 
- From the data reported by Bédoui et al. (2006), the Young's moduli of the crystalline lamellae are determined in the main directions, as being $E_{c x x}=6.3 \mathrm{GPa}, E_{c y y}=9.5 \mathrm{GPa}$, and $E_{c z z}=40.2 \mathrm{GPa}$.

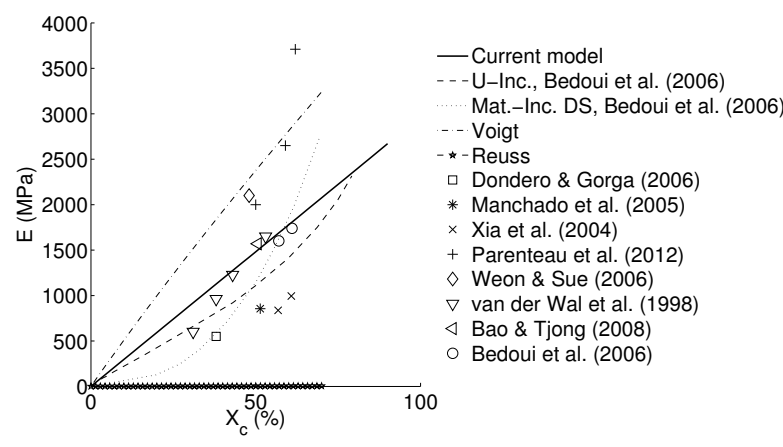

(a)

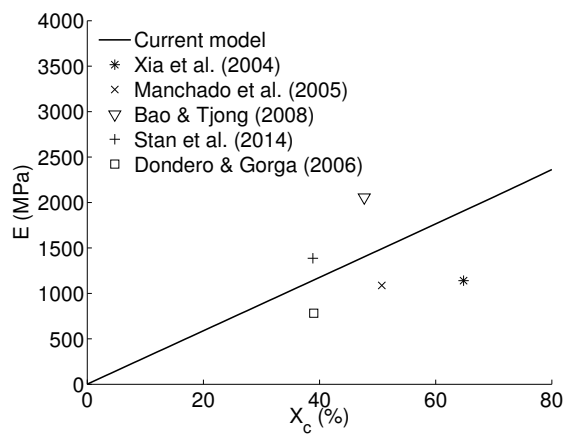

(b)

Figure 10: Theoretical models predictions in comparison with experimental results from the literature: (a) developed model and literature models for neat PP compared to the experimental results from Dondero and Gorga (2006); Manchado et al. (2005); Xia et al. (2004); Parenteau et al. (2012); Weon and Sue (2006); van der Wal et al. (1998); Bao and Tjong (2008); Bédoui et al. (2006) and (b) developed model for 1\%wt CNTs PP compared to the experimental results from Stan et al. (2014); Bao and Tjong (2008); Manchado et al. (2005); Xia et al. (2004); Dondero and Gorga (2006).

Figure 10a shows the results of the current theoretical mode 4 in comparison with experiments from literature in the case of neat PP and for various degrees of crystallinity. The developed model predictions are also compared to the so-called Matrix-Inclusion dilute scheme method (using aspect ratios of 10/1.2 and 100/1.2 for respectively the width and length over the thickness) (van Dommelen et al. 2003; Bédoui et al., 2006), to the so-called U-inclusion model (van Dommelen et al., 2003; Bédoui et al., 2006), and to the Reuss and Voigt bounds. Although the different PP samples used can have different polymer structures, the current model provides a prediction of the Young's modulus in terms of the degree of crystallinity as good as the other two models, while remaining simpler in its implementation. Figure 10p compares the current theoretical model results to experimental results

\footnotetext{
${ }^{4}$ For low crystallinity and low CNTs content, the Young modulus is close to $E_{m}=0.9$ $\mathrm{MPa}$, which explains why it almost vanishes on the ordinate axis.
} 
from the literature in the case of PP reinforced by $1 \%$ wt CNTs. A good agreement is also observed.

3.3. Comparison of the theoretical model predictions with experimental results for the processed nanocomposite material

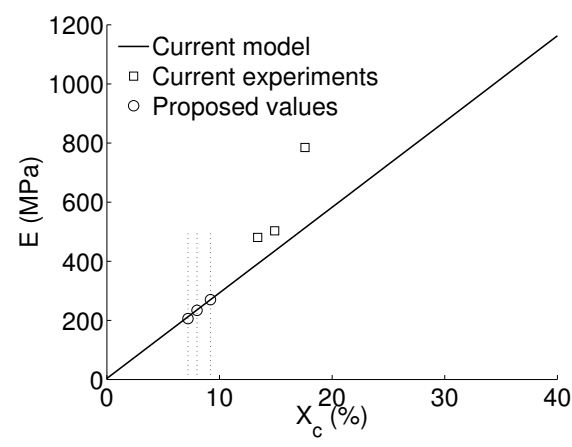

(a) $4 \%$ wt CNTs

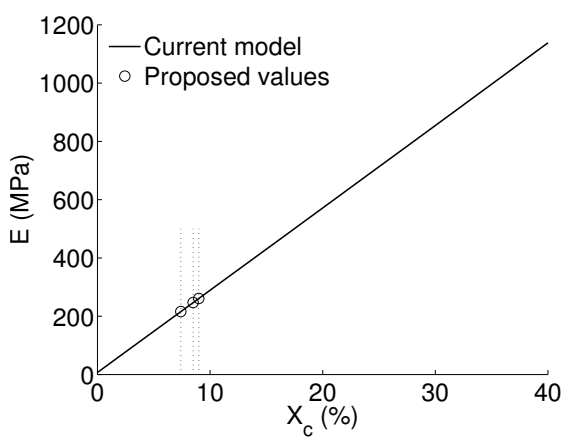

(b) $8 \%$ wt CNTs

Figure 11: Current theoretical results for the $4 \%$ wt and $8 \%$ wt PP/CNTs nanocomposite materials in comparison with the experimental results reported in Tab. 3. The proposed values refer to the extrapolated Young's modulus for the cell walls of the foamed samples, based on their crystallinity degree.

Figure 11a compares the model predictions with the experimental results obtained on the three-point bending tests for the $4 \% \mathrm{wt}$ PP/CNTs nanocomposite material, see Tab. 3. It can be seen that comparable results are obtained. The theoretical results for $8 \%$ wt PP/CNTs nanocomposite material is illustrated in Fig. 11b.

Since it is very difficult to measure the modulus of the cell walls of the foamed samples, this value is reasonably estimated via theirs actual degree of crystallinity from Figs. 11a and 11b, for the foamed samples with $4 \% \mathrm{wt}$ CNTs and $8 \%$ wt CNTs, respectively. The resulting values are reported in Tab. 4 and they will be used in the next section to study the foamed material behavior from a unit cell model.

\section{Micro-macro homogenization: Unit cell computational micro- mechanics model}

The computational micro-mechanics approach is frequently used to model structures made of heterogeneous materials. This approach is herein adopted 
Table 4: Extrapolated Young's modulus for the cell walls material from Fig. 11 for the different foamed samples (refer to Fig. 2 for details of the foaming conditions).

\begin{tabular}{lcc} 
Sample code & $X_{c}(\%)$ & $E_{s}(\mathrm{MPa})$ \\
\hline NC4F1 & 8.0 & 234 \\
\hline NC4F2 & 9.2 & 270 \\
\hline NC4F3 & 7.2 & 206 \\
\hline NC8F1 & 8.5 & 247 \\
\hline NC8F2 & 9.0 & 261 \\
\hline NC8F3 & 7.4 & 216 \\
\hline
\end{tabular}

in order to estimate the homogenized properties of nanocomposite foams. Its basic principle and its application to foamed materials through unit cell models with imperfections are described in this section.

\subsection{Computational micro-mechanics method}

The basic idea of the computational micro-mechanics method is to estimate the homogenized properties of heterogeneous materials based on a finite element model of the characteristic volume element of the underlying micro-structure, see (Peric et al., 2010) for details. This characteristic volume element should be statistically representative and leads to the use of a representative volume element (RVE). For periodic structures, under the assumption of the periodic local field, the RVE can be selected as the repeated unit since this RVE contains all necessary information about the micro-structure. For random structures, the RVEs are defined in a statistical way (Kanit et al., 2003). In all generalities, the relation between the homogenized field $f$ and the local field $f$ is expressed under the form of the volume integral (so-called homogenization) over the RVE as

$$
\bar{f}=<f>=\frac{1}{V} \int_{V} f(\mathbf{x}) d V
$$

where $V$ is the volume of the considered RVE and where $\mathbf{x}$ stands for the local position vector. Equation (14) implies that the local inhomogeneous field is considered as homogeneous under an average sense at the structural scale.

For a linear elastic problem, the Hooke matrix of the homogenized medium can be explicitly estimated. The material constants as the Young's modulus $E$, Poisson ratio $\nu$, shear modulus $G$, etc. can then be determined. For 
non-linear and path-dependent problems (e.g. large strain, plasticity, strain localization), the homogenization process yields a macro-stress vs. macrostrain relation, $\overline{\boldsymbol{\sigma}}-\overline{\boldsymbol{\varepsilon}}$, which is obtained from the (iterative) resolution of the micro-mechanics boundary value problem (BVP). In this case, the (macro)stress-strain constitutive law cannot be expressed by an explicit relation. However, as the stress-strain energy relation always exists in an implicit form via the resolution of the microscopic BVP, it is possible to solve the problem of a macro-scale structure with a two-scale approach even when studying micro-instabilities as shown by Nguyen and Noels (2014). To this end the periodic boundary condition (PBC) to be applied on the microscopic BVP has been suitably formulated by Nguyen and Noels (2014) to capture the buckling behavior.

\subsection{Unit cell models for closed cell foams}

The definition of the RVE is an important ingredient of the computational micro-mechanics model to correctly capture the homogenized behavior of the foamed material. For periodic structures, the RVE can be chosen as the repeated unit pattern. For random structures, the RVE is defined in a statistical way with a larger number of realizations for each RVE size in order to capture all the possible micro-structure arrangements. Although the real foamed materials possess a large range of cell sizes and of cell shapes, regular arrangement models are still considered because of their efficiency and simple implementation. With this assumption, a whole cellular structure is represented by the space-filling of a repeated pattern following the periodicity vectors. The study of whole structure is then performed on the related repeated pattern (including one or several unit cells), completed with the periodic boundary condition (Nguyen et al. 2012 ) to ensure the periodicity the local fields.

Some unit cell models are available in the literature as a truncated cube model (Santosa and Wierzbicki, 1998), a cruciform-hemisphere model (Meguid et al. 2002), a hollow model (Czekanski et al., 2005), a tetrakaidecahedron model (Simone and Gibson, 1998a), etc. Simone and Gibson (1998a) have shown that the tetrakaidecahedron unit cell, see Fig. 12 a, has a relatively low anisotropy and is thought to be a good choice for capturing the homogenized behavior of macroscopically isotropic foamed materials, as it is the case with the studied foams. Thus this unit cell model is adopted in this work. The degenerate case of open cell, which is generated from a ideal closed unit cell by removing all faces, see Fig. $12 \mathrm{~b}$, is also studied. 


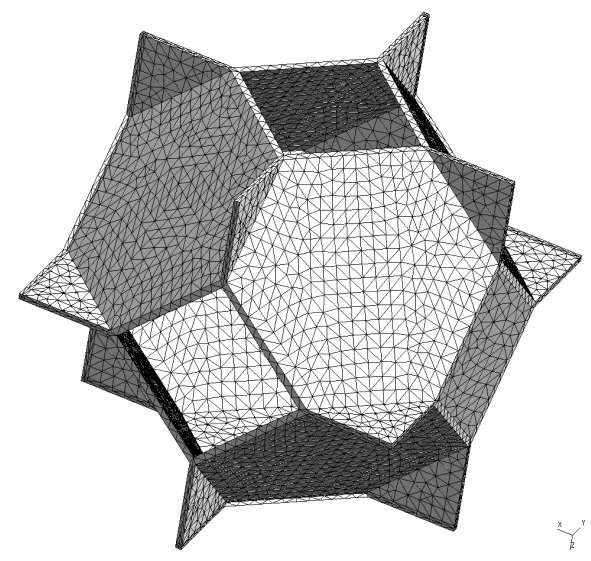

(a) Ideal closed unit cell

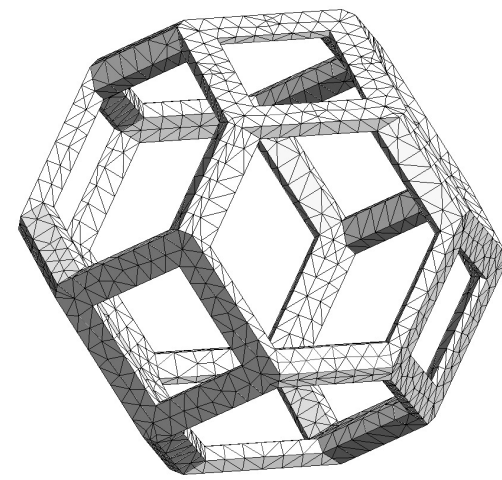

(b) Open unit cell

Figure 12: Tetrakaidecahedron unit cell with relative density $\rho / \rho_{s}=0.05$ for an ideal closed unit cell (a) with constant thickness and for an open unit cell (b).

The most important parameter of the foamed materials is the relative density, which is easily measured. However, the use of "perfect" unit cell models usually leads to an overestimation of the stiffness. Indeed, the real foams contain a large number of imperfections, which include random cell sizes and cell shapes, cell wall thickness variations, cell wall curvatures, missing cell walls, cell wall corrugations, cell wall fractures, etc., see the real micro-structure images in Fig. 3. Different kinds of imperfections in cellular structures have been studied by Grenestedt (2005), who have shown their large effect on the foam mechanical behavior.

The unit cell models can take into account several imperfections under the assumption that they correspond to periodic distributions. In this work, we consider the solid concentration at the junctions of cell faces (so-called Plateau border) (Simone and Gibson, 1998a), the cell wall curvature, the cell wall waviness (Simone and Gibson, 1998b), as well as their combination. Their effects on the homogenized compressive response are successively studied after the description of the numerical models.

\subsubsection{Unit cell model with solid concentration at the cell face junctions (so- called Plateau border)}

The solid concentration at the cell face junctions often appears in cases of closed cell foams with thin cell walls. With this model, a percentage of solids 


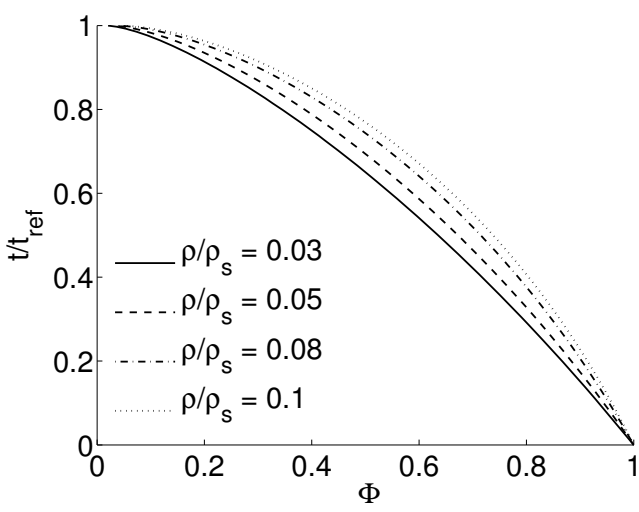

(a) Cell wall thickness versus $\Phi$

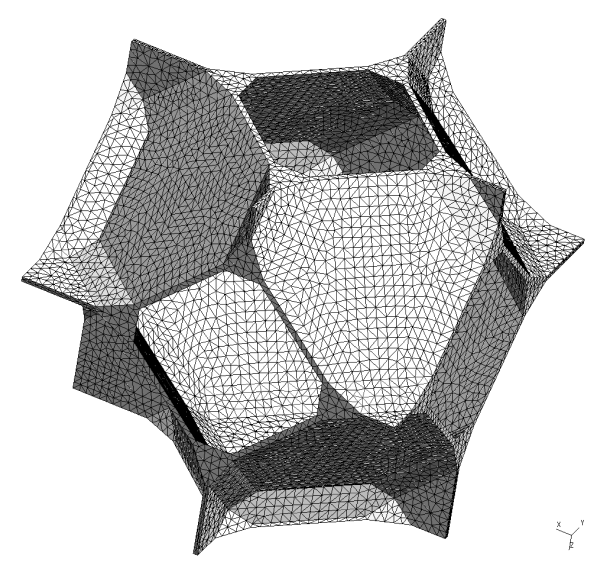

(b) $\Phi=0.6$ and $\rho / \rho_{s}=0.05$

Figure 13: Reduction of cell face thickness when increasing the Plateau border parameter $\Phi$ (a) and a closed tetrakaidecahedron unit cell characterized by $\Phi=0.6$ and $\rho / \rho_{s}=0.05$ (b). For each value of $\rho / \rho_{s}, t_{\text {ref }}$ is the thickness of the cell face of the closed unit cell without considering the Plateau border.

is concentrated at the junctions of the cell faces and this concentration is represented by the factor $\Phi$. This factor is defined as the ratio of the density of an open cell foam obtained by removing all the cell faces of the respective closed cell foam and the density of this closed cell foam. By modifying the solid distribution in that way, the mechanical stiffness is modified when increasing the value of $\Phi$ and degenerates to the case of open cell foams when $\Phi=1$. The measurement of $\Phi$ for rigid polyurethane foams performed by Reitz et al. (1984) typically leads to a value of 0.8. For PMMA foams, Chen et al. (2011) have used a value equal to 0.9 .

When considering the Plateau border imperfection, the homogenized properties depend on both $\Phi$ and on the relative density $\rho / \rho_{s}$. The cell face thickness of the unit cell is estimated from the values of $\Phi$ and of $\rho / \rho_{s}$. The dependency of the cell face thickness on $\Phi$ is depicted in Fig. [13a for several values of $\rho / \rho_{s}$. When $\Phi$ increases, the cell face thickness deceases. When $\Phi=1$, an open unit cell is obtained since the cell face thickness vanishes. Figure $13 \mathrm{~b}$ illustrates the resulting tetrakaidecahedron unit cell characterized by a relative density $\rho / \rho_{s}=0.05$ and $\Phi=0.6$. 


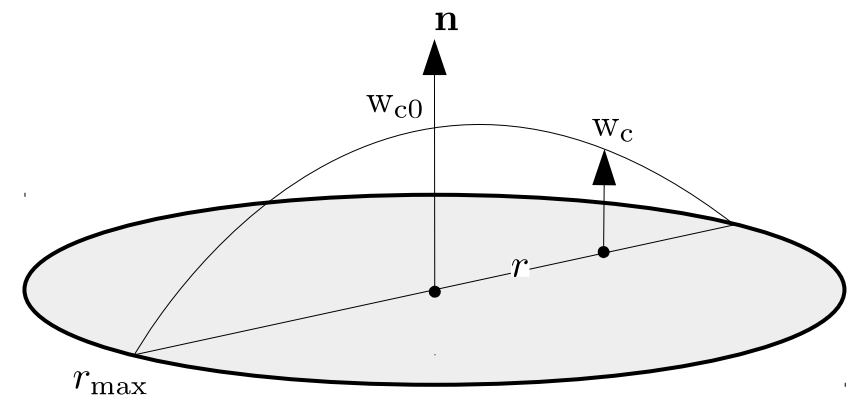

Figure 14: Axial coordinate system $\left(r, \mathrm{w}_{c}\right)$ embedded in a face with the outward normal n. In this figure, $r$ is the distance from an arbitrary point of the face to its center.

\subsubsection{Unit cell model with cell face curvature}

The stress induced during the foaming process can result into curvature of the obtained foam cell faces, see real micro-structure images in Fig. 3. Thus the influence of the cell face curvature needs to be considered: the cell face curvature is constructed by moving both hexagonal and square faces of an ideal tetrakaidecahedron closed unit cell following their original outward unit normal $\mathbf{n}$ so that each curved face becomes a spherical cap with a maximal deflection $\mathrm{w}_{c 0}$ at its center, see Fig. 14. For an initially planar face with an embedded axial coordinate system $\left(r, \mathrm{w}_{c}\right)$ linked to its center, the spherical cap is expressed as

$$
\frac{\mathrm{w}_{c}}{\mathrm{w}_{c 0}}(r)=\frac{1}{\lambda} \sqrt{\frac{1}{4}\left(\lambda+\frac{1}{\lambda}\right)^{2}-\left(\frac{r}{r_{\max }}\right)^{2}}-\frac{1}{2}\left(\frac{1}{\lambda^{2}}-1\right),
$$

where $r_{\max }$ is the maximal perturbed radius, $\mathrm{w}_{c 0}$ is the maximal out-of-plane deflection at the face center, and where $\lambda$ is the shape factor defined by

$$
\lambda=\frac{\mathrm{w}_{c 0}}{r_{\max }} .
$$

With the introduction of the cell face curvature, the homogenized properties depend on the relative density $\rho / \rho_{s}$, but also on the face curvature, which is characterized by $\lambda$ and $r_{\max }$. If the maximal perturbed radius $r_{\max }$ remains constant for each face of the ideal closed unit cell, the curvature of each face is only controlled by the shape factor $\lambda$. In this case, the influence of the shape ratio $\lambda$ on the shape of a spherical cap is shown in Fig. 15a. 


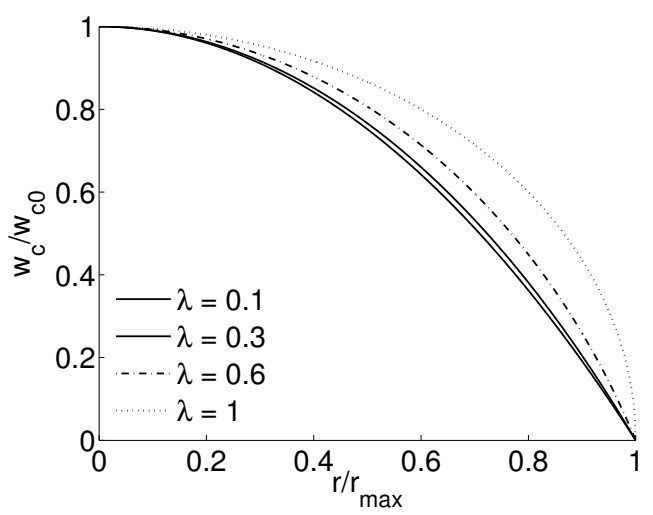

(a)

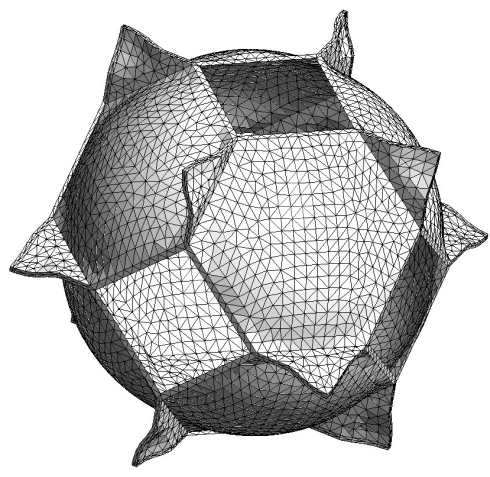

(b) $\lambda=0.33$ and $\rho / \rho_{s}=0.05$

Figure 15: Influence of the shape factor $\lambda$ on the cell face geometry (a), and a curved closed unit cell with $\rho / \rho_{s}=0.05$ and with $\lambda=0.33$ for both the hexagonal and square faces.

A plane face corresponds to $\lambda=0$ and an hemisphere correspond to $\lambda=1$. Figure 15b depicts a closed unit cell with $\rho / \rho_{s}=0.05$ and with a cell face curvature characterized by $\lambda=0.33$ for both the hexagonal and square faces.

\subsubsection{Unit cell model with cell face waviness}

The cell faces of a cellular structure can be corrugated due to the stress induced during the foaming process. This cell face corrugation is herein modeled by a waviness imperfection of the hexagonal cell faces.

Using the coordinate system $\left(r, h, \mathrm{w}_{\text {wave }}\right)$ embedded in an initially planar face, see Fig. 16, the cell face waviness is modeled by combining a sinus function of the signed distance $h$ (which can be positive or negative) to a spherical cap governed by Eq. (15), leading to

$$
\mathrm{w}_{\text {wave }}(h, r)=\sin \left(\theta \frac{\pi h}{r_{\max }}\right) \mathrm{w}_{c}(r),
$$

where $r_{\max }$ is the maximal perturbed radius, $\mathrm{w}_{c}(r)$ is given by Eq. (15), and where $\theta$ is defined as the waviness period number.

With the introduction of the cell face waviness, the homogenized properties depend on the relative density $\rho / \rho_{s}$ and on the face waviness, which is characterized by $\lambda, r_{\max }$, and $\theta$. The dependency of the wavy faces on the 


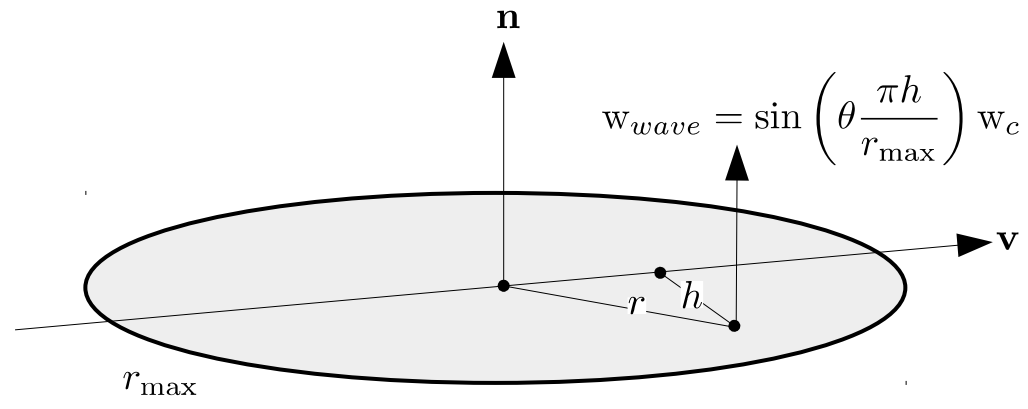

Figure 16: Coordinate system $\left(r, h, \mathrm{w}_{\text {wave }}\right)$ used to generate the cell face waviness. In this figure, $\mathbf{n}$ is the outward normal to the planar face, $\mathbf{v}$ is the direction perpendicular to the waviness propagation, $r$ is the distance from an arbitrary point on the face to its center and $h$ is the signed distance from this point to the vector $\mathbf{v}$.

waviness period number $\theta$ at the cross section $h= \pm r$ is depicted in Fig. 17a. A planar face corresponds to $\theta=0$. Figure 17b illustrates a closed unit cell with $\rho / \rho_{s}=0.05$ and with a cell face waviness characterized by $\theta=3$ and by $\lambda=0.17$ applied to all hexagonal faces.

\subsubsection{Unit cell model with imperfections combination}

The different imperfection types present in the real foamed structure can be combined in the unit cell model under the assumption of their periodicity. Therefore, the Plateau border, the cell face curvature, and cell face waviness imperfections described above can be simultaneously applied on a ideal tetrakaidecahedron closed unit cell model.

Figure 18 depicts a closed unit cell with $\rho / \rho_{s}=0.065$ (same relative density as for the NC4F1 samples) with such a combination. The plateau border is characterized by $\Phi=0.6$, hexagonal faces waviness by $\theta=3$ and a propagation direction along the $x$-axis, and the faces curvature by $\lambda=0.17$.

\subsection{Material models for cell walls}

To estimate the homogenized Young's modulus of the foamed samples by using the computational micro-mechanics model, the cell walls are assumed to obey an elastic law characterized by an elastic modulus $\mathrm{E}_{s}$ and a Poisson ratio $\nu_{s}$. The value of $\mathrm{E}_{s}$ is reported in Tab. 4, as discussed in the previous section, and the value of $\nu_{s}$ is equal to 0.45 , which is known as the typical value for low crystallinity polypropylene. 


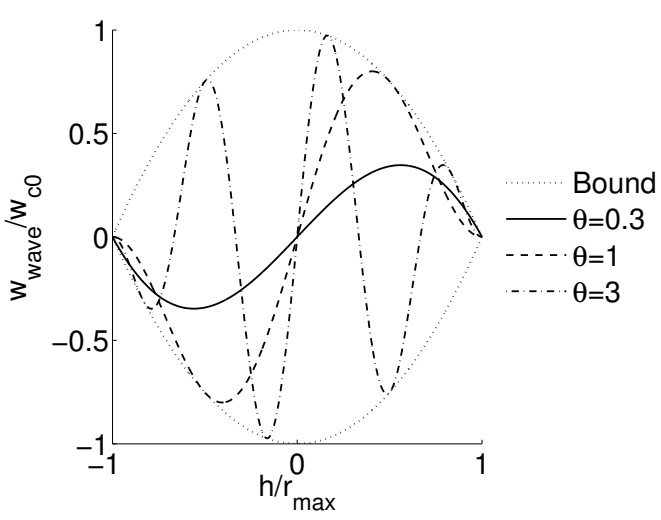

(a)

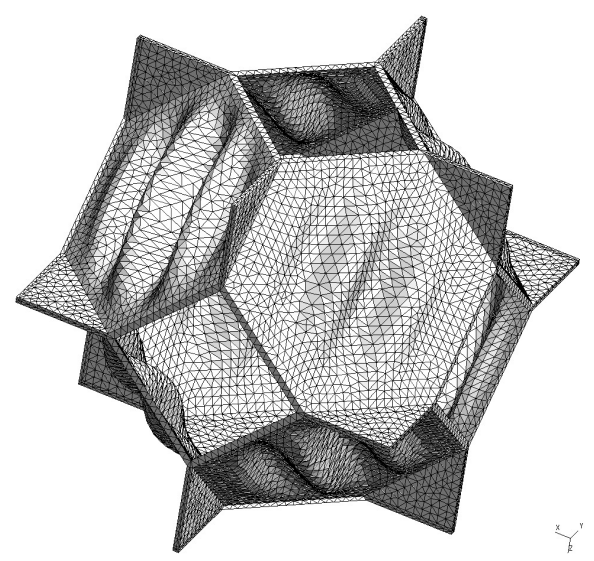

(b) $\theta=3, \lambda=0.17$ and $\rho / \rho_{s}=0.05$

Figure 17: Cross section $h= \pm r$ for different values of the waviness period $\theta$ (a) and a closed unit cell with $\rho / \rho_{s}=0.05, \theta=3$, and $\lambda=0.17$ with the waviness following the $x$-axis (b).

Since the foamed samples have a crystallinity degree lower than $10 \%$, see Tab. 2, the elastic collapse mode is preferred to the plastic collapse mode to model the plateau occurring under compressive loading (Gibson and Ashby, 1997, e.g.). Moreover, the cell walls are assumed to obey to the rate independent elastic large strain Neo-Hookean constitutive law detailed in Appendix A. The rate-independence assumption will be discussed later in this section.

\section{Numerical results and discussions}

In this section, the compressive curves of the foamed material are obtained using the tetrakaidecahedron unit cell-based micro-mechanics approach described in Section 4. The obtained numerical results are compared to the experimental results provided in Section 2. First the linear responses are compared to discuss the influence of the imperfections on the homogenized Young's modulus of the foamed material. Then the full compressive curves, including the plateau stage, are considered in order to demonstrate the ability of the method to capture the non-linear large deformation behavior. 


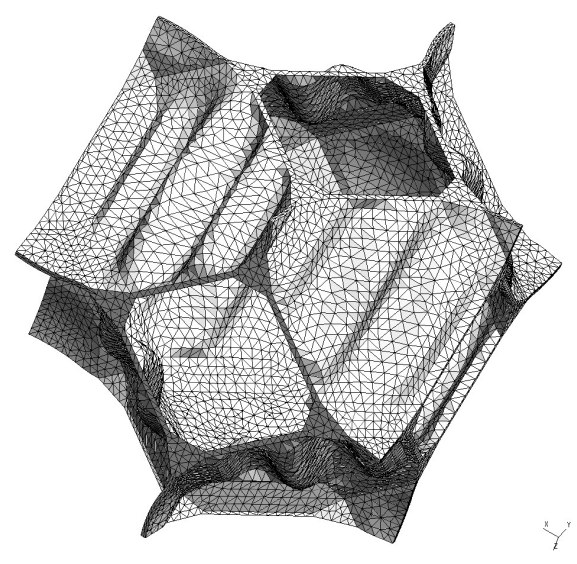

Figure 18: A closed unit cell with $\rho / \rho_{s}=0.065$ combining the different imperfections: the plateau border is characterized by $\Phi=0.6$, hexagonal faces waviness by $\theta=3$ and a propagation direction along the $x$-axis, and the faces curvature by $\lambda=0.17$.

\subsection{Homogenized Young's modulus using the computational micro-mechanics approach}

\subsubsection{Ideal unit cell model}

Results obtained with the ideal closed unit cell model developed by $\mathrm{Si}$ mone and Gibson (1998a) and with the open cell model developed by Zhu et al. (1997) are reported in Fig. 19 to verify the implemented computational approach. Moreover, the figure compares the experimental results of the foamed samples, see Fig. 7, to the predictions obtained with the tetrakaidecahedron unit cell model for both the open cell case $(\Phi=1)$ and the closed cell case with constant cell wall thickness. As mentioned in the previous section, on the one hand, the ideal tetrakaidecahedron unit cell model leads to an overestimation of the real modulus, and on the other hand, the experimental results are larger than the results given with the open cell model for the same foam density, motivating the introduction of imperfections in the ideal unit cell model.

\subsubsection{Unit cell model with Plateau border}

Figure 20a illustrates the influence of the mass concentration factor $\Phi$, see Section 4.2.1, on the homogenized Young's modulus. When the value of $\Phi$ increases, the obtained Young's modulus progressively changes from the value of the closed cell model to the value of the open cell model, at constant relative density. If a high value of $\Phi$ is used, e.g. $\Phi=0.8$, a large reduction 


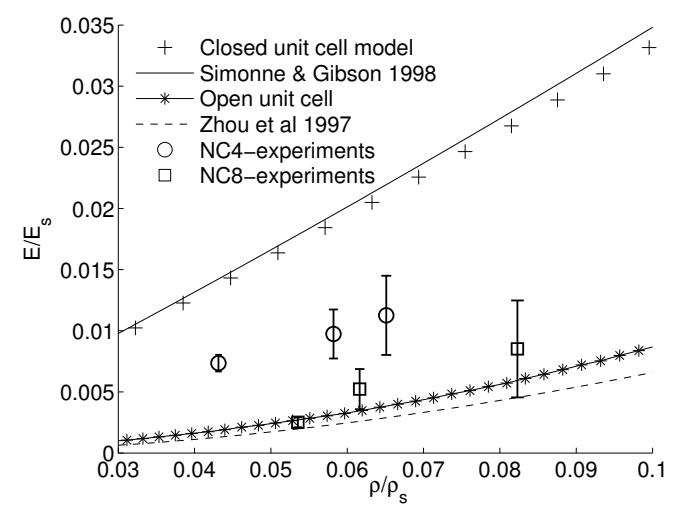

Figure 19: Prediction of the relative initial Young's modulus of the foamed samples (denoted by $E / E_{s}$ ): Comparison of the closed cell (without considering $\Phi$ ) and open cell $(\Phi=1)$ results of the developed model with the tetrakaidecahedron closed cell model developed by Simone and Gibson (1998a) and the tetrakaidecahedron open cell model developed by Zhu et al. (1997). The experimental Young's modulus measurements are also reported.

of the homogenized Young's modulus is observed. This is explained as, in this case, the material moves from the cell faces to the cell face junctions. The Young's modulus of the closed cell foams combines the effects from the cell face stretching and from the cell edge bending. However, the reduction of the stiffness of the cell face stretching is dominant for closed cell foams, leading to a reduction of the homogenized Young's modulus.

The influence of the concentration factor $\Phi$ on the predicted homogenized Young's modulus of the foamed materials is depicted in Fig. 20b in terms of the relative density. The average experimental results are also reported. For all the $4 \%$ wt CNTs foamed samples, the average value of the experimental results is comparable with the results of the computational unit-cell model for values of $\Phi$ in between 0.85 and 0.95 . All the $8 \%$ wt CNTs foamed samples require larger values of $\Phi$-in between 0.95 and 1 - to predict the correct behavior. We known that the unit cell model works under the assumption of the periodic micro-structure arrangement. When the CNT content and the soaking temperature are increased, the cell disorder and imperfections are also increased so that the use of the unit cell model becomes less appropriate. Although modeling these imperfections with a high mass concentration factor remains possible, it is more relevant to combine the unit cell model with other imperfections as cell face curvature and cell face waviness. As it will 


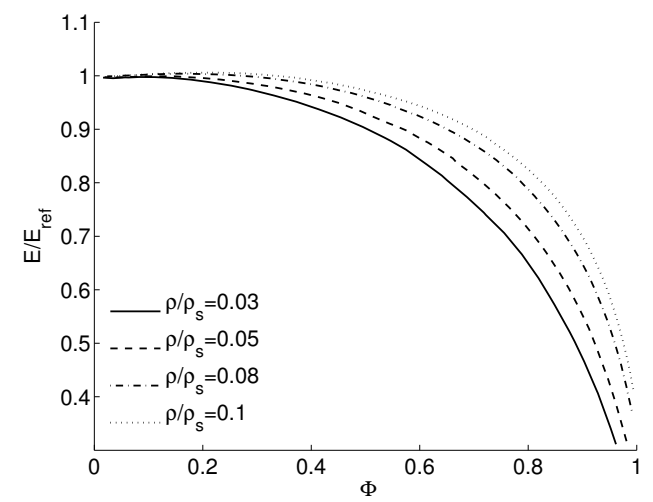

(a) Effect of $\Phi$

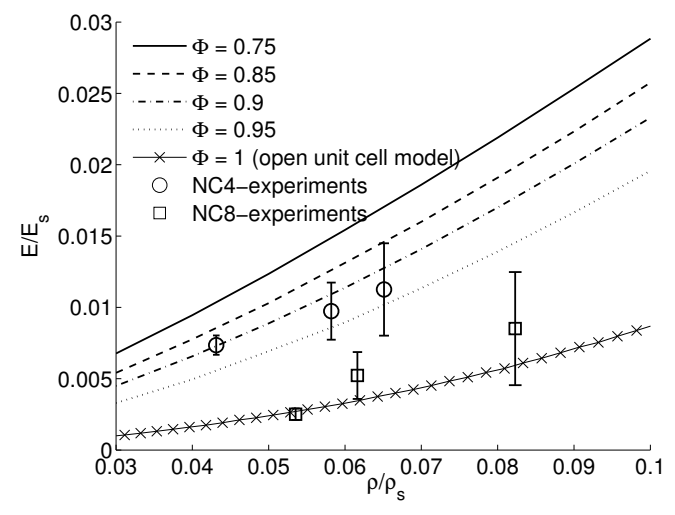

(b) Comparison with experiments

Figure 20: Influence of the mass concentration factor $\Phi$. (a) Effect of the mass concentration factor $\Phi$ on the homogenized Young's modulus $-E$ and $E_{\text {ref }}$ are the Young's modulus of the unit cell model for different values of $\Phi$ and of the closed unit cell model with constant cell wall thickness, respectively. (b) Comparison of the predicted homogenized elastic modulus for different mass concentration factors $\Phi$ and experimental results of the current foamed samples.

be shown, this allows a smaller value of $\Phi$ to be considered in order to predict the correct homogenized properties of the foamed material.

\subsubsection{Unit cell model with cell face curvature}

The curved unit cell model described in Section 4.2 .2 is considered using the same shape factor $\lambda$ for both the hexagonal and square faces. As the value $r_{\max }$ is kept constant, the homogenized Young's modulus depends on the shape factor $\lambda$ and on the relative density $\rho / \rho_{s}$, only.

Figure 21a shows the influence of the shape factor $\lambda$ on the homogenized elastic modulus for different values of $\rho / \rho_{s}$. It can be seen that an increase of the shape factor leads to a reduction of the homogenized Young's modulus. This point is explained by the significant reduction of both the axial stiffness and flexural rigidity of the cell faces resulting from the increase of the cell face curvature. This effect is more pronounced at the low relative densities.

The influence of the shape factor $\lambda$ on the predicted homogenized Young's modulus of the foamed materials is illustrated in Fig. 21b in terms of the relative density. The experimental results are also reported on the same figure. Although values of $\lambda$ between 0.3 and 0.5 can be used to capture the average results for the $4 \%$ wt CNTs foamed samples, the $8 \%$ wt CNTs foamed samples would require a larger value of $\lambda(\lambda>0.5)$ to be correctly 


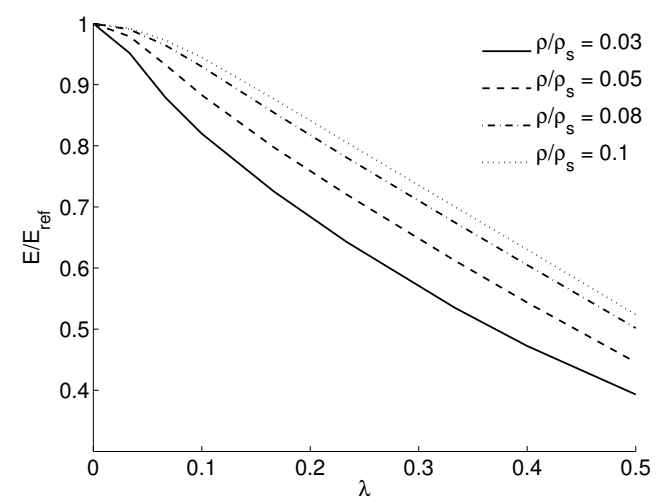

(a) Effect of $\lambda$

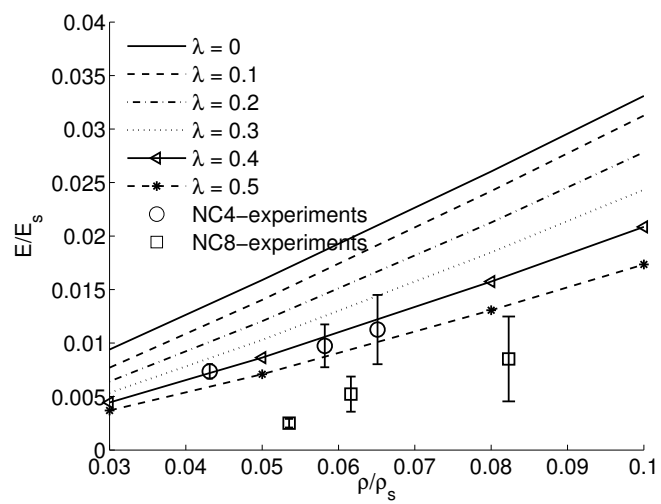

(b) Comparison with experiments

Figure 21: Influence of the shape factor $\lambda$. (a) Effect of the shape factor $\lambda$ on the homogenized Young's modulus $-E$ and $E_{\text {ref }}$ are the Young's modulus of the unit cell model for different values of $\lambda$ and of the closed unit cell model with $\lambda=0$, respectively. (b) Comparison of the predicted homogenized elastic modulus for different shape factors $\lambda$ and experimental results of the current foamed samples.

modeled. However, it is more appropriate to combine the imperfections.

\subsubsection{Unit cell model with cell face waviness}

The cell face waviness is added on the hexagonal faces of the tetrakaidecahedron. By using a fixed spherical cap characterized by $\lambda=0.17$. The homogenized Young's modulus depends on the number $\theta$ of the waviness period and on the relative density $\rho / \rho_{s}$.

Figure $22 \mathrm{a}$ reports the influence of the face waviness period number $\theta$ on the homogenized elastic modulus for different values of $\rho / \rho_{s}$. It is clear that the homogenized Young's modulus is reduced when increasing the number of the face waviness period. For small values of $\theta(\theta \leq 3)$, the cell face waviness leads to a significant reduction of both the axial stiffness and flexural rigidity and, as a result, of the homogenized Young's modulus. When $\theta$ becomes larger, $\theta>3$, the homogenized modulus is less sensitive to the waviness period number, and even starts to increase slowly when $\theta>4$. Once more, the effects are more important for smaller relative densities.

The effect of the shape ratio $\lambda$, at constant face waviness period number, $\theta=1$, is depicted in Fig. $22 \mathrm{~b}$ for a relative foam density of 0.065 which corresponds to the NC4F1 case. The experimental results of the NC4F1 samples are also reported. This figure shows that the homogenized Young's 


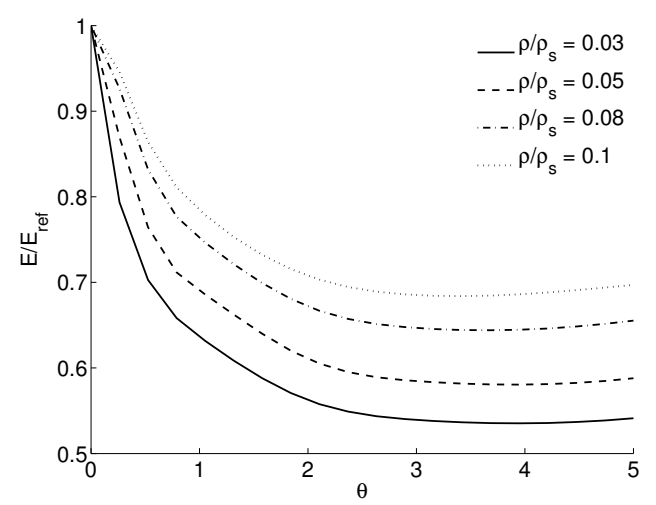

(a) Effect of $\theta$

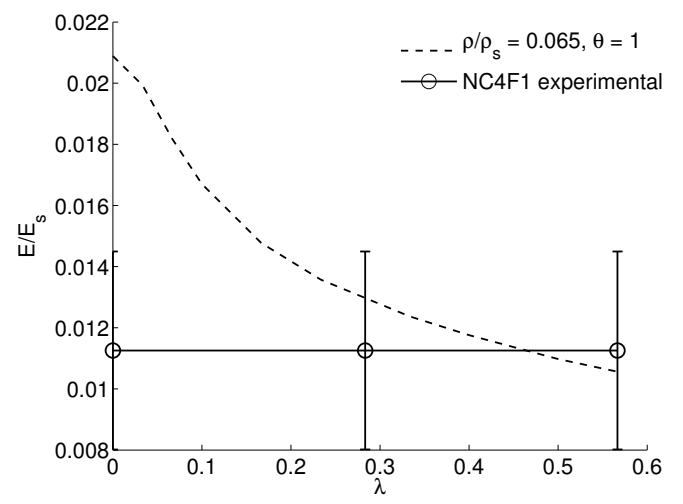

(b) Effect of $\lambda$

Figure 22: Influence of the waviness period $\theta$ number. (a) Effect of the waviness period $\theta$ on the homogenized Young's modulus $-E$ and $E_{\text {ref }}$ are the Young's modulus of the unit cell model for different values of $\theta$ and of the closed unit cell model with $\theta=0$, respectively. (b) Effect of $\lambda$ at constant $\theta=1$ for the NC4F1 case.

modulus can be correctly captured by using a suitable value of $\theta$ and $\lambda$.

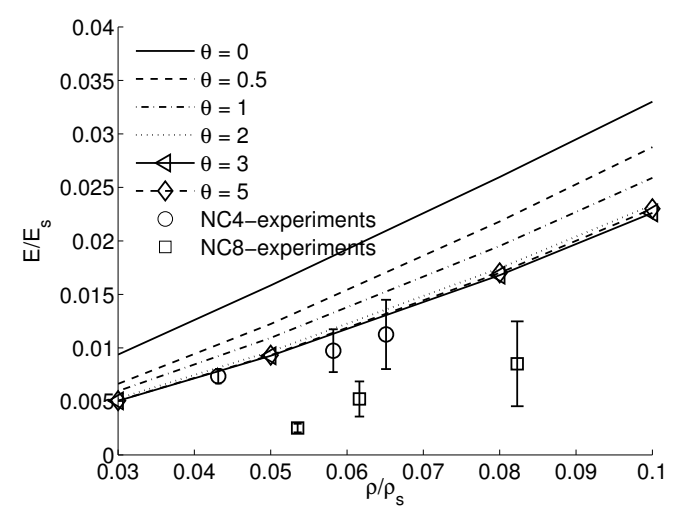

Figure 23: Comparison of the predicted homogenized elastic modulus for different values of $\theta$ at constant value of $\lambda=0.17$ to the experimental results.

The effect of the waviness period number $\theta$ on the predicted homogenized Young's modulus of the foamed materials is show in Fig. 23 in terms of the relative density and for a constant value of $\lambda=0.17$. The experimental results are also reported. In this case, all values of $\theta$ overestimate the experimental results. To predict correctly the homogenized modulus, a larger value of $\lambda$ should be used as demonstrated in Fig. 22 b. Although all values 
of $\theta$ and $\lambda$ can possibly be used, in the next paragraph the combination of the imperfections is investigated.

\subsubsection{Unit cell model with imperfections combination}

As demonstrated in the previous paragraphs, each imperfection type (Plateau border, cell face curvature, or cell face waviness) cannot be separately used with suitable parameters in order to accurately capture the homogenized Young's modulus, unless the imperfection parameters are taken at the higher end. These imperfections should thus be treated in a combined form.

The homogenized Young's modulus of the unit cell model is studied for the following combinations of imperfections:

- A unit cell model with a relative density $\rho / \rho_{s}=0.065$, corresponding to the NC4F1 foam samples, different Plateau border $\Phi$ values, square face curvature characterized by $\lambda=0.17$, and hexagonal face waviness characterized by $\lambda=0.17$ and $\theta=3$;

- A unit cell model with a relative density $\rho / \rho_{s}=0.065$, corresponding to the NC4F1 foam samples, different Plateau border $\Phi$ values, square face curvature characterized by $\lambda=0.28$, and hexagonal face waviness characterized by $\lambda=0.28$ and $\theta=1$; and

- A unit cell model with a relative density $\rho / \rho_{s}=0.082$, corresponding to the NC8F1 foam samples, different Plateau border $\Phi$ values, square face curvature characterized by $\lambda=0.5$, and hexagonal face waviness characterized by $\lambda=0.5$ and $\theta=3$.

The dependency of the homogenized Young's modulus on $\Phi$ is reported in Fig. 24a for two combinations of imperfections. It can be seen that the two curves are almost similar. The obtained results are compared to the experimental results in Fig. 24b. The results with the ideal closed unit cell and open unit cell are also reported, and it can be seen that the homogenized Young's moduli are upper-bounded by the ideal closed unit cell and lower-bounded by the open unit cell. When the Plateau border is used in combination with the square face curvature and with the hexagonal face waviness, comparable results are obtained in a large range of $\Phi$ values (for $\Phi<0.8)$.

Figure 25 compares the predicted homogenized Young's modulus for different Plateau border parameters $\Phi$ with the experimental results of the 


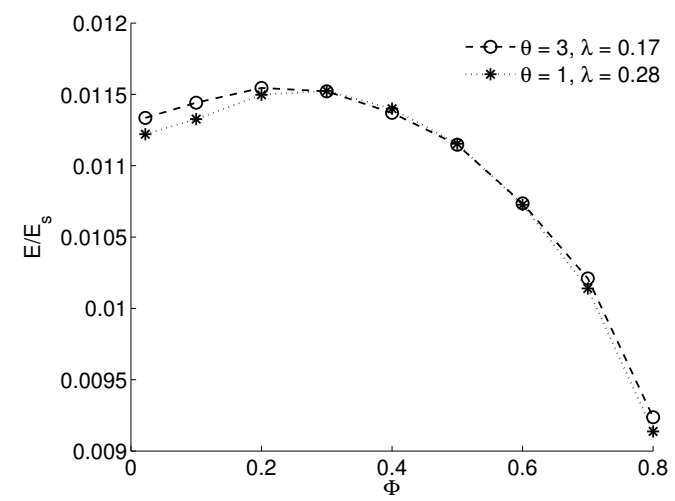

(a) Imperfections combination

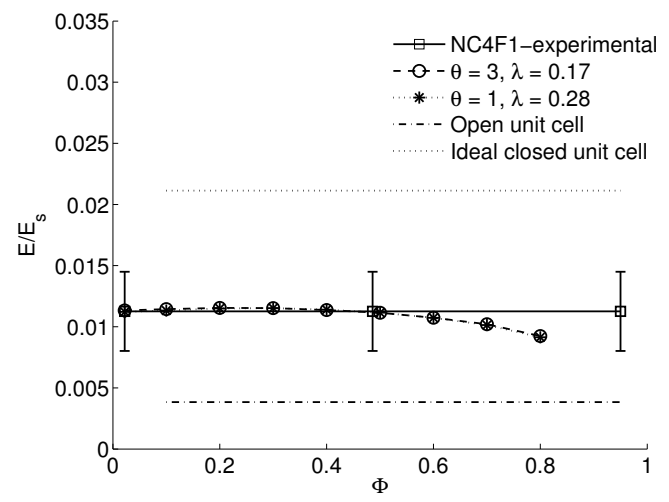

(b) Comparison with experiments

Figure 24: Homogenized Young's modulus of the NC4F1 foam. (a) Predictions with different combinations of imperfections in terms of the Plateau border parameter $\Phi$. (b) Comparison with experimental results.

NC8F1 foamed samples. The results of the ideal closed unit cell and open unit cell with the same relative density are also reported. As for the NC4F1 foamed samples, the homogenized Young's moduli are upper-bounded by the ideal closed unit cell and lower-bounded by the open unit cell, while good predictions are obtained with the combination of imperfection for a large range of values of $\Phi(\Phi<0.8)$.

\subsection{Large-strain uniaxial compression behavior using a computational micro- mechanics approach}

Since the considered foamed samples possess a relatively low degree of crystallinity (lower than 10\%, see Tab. 2) the collapse mode of the foamed material results from the elastic buckling of the cell walls, Gibson and Ashby, 1997, e.g.), and the unit-cell compressive curves do not exhibit a strain softening part. Moreover, the buckling mode is defined by the imperfections combination and the unit-cell response does not exhibit instabilities, snapback, nor bifurcation. Hence, a unit cell model can be used with appropriate periodic boundary conditions Nguyen and Noels (2014), to predict the compressive response of the foamed material.

The compressive stress-strain curves obtained with the elastic large strain Neo-Hookean constitutive model are reported in Fig. 26 for a relative density of 0.66 corresponding to the NC4F1 samples and for the different mass concentration factors $\Phi$ of $0.4,0.6,0.7$, and 0.8 . The numerical tests are 


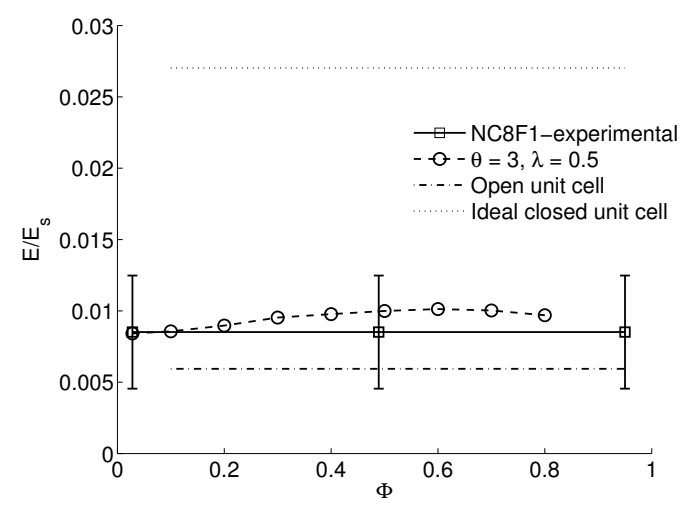

Figure 25: Homogenized Young's modulus of the NC8F1 foam: predictions with a combination of imperfections in terms of the Plateau border parameter $\Phi$ and comparison with experimental results.

performed up to the point for which self contact of cell walls occurs as the contact phenomenon is not accounted for in this work. It can be seen that the experimental results are close to the numerical prediction obtained for the different values of $\Phi$. Figure 27 illustrates at different compression stages of the unit cells with $\Phi=0.6$ the distribution of the local relative equivalent strain rate, $\dot{\varepsilon}_{\text {rel }}$, defined by

$$
\dot{\varepsilon}_{\text {rel }}=\frac{\dot{E}_{\mathrm{eq}}}{\dot{\bar{F}}_{x x}}
$$

where $\dot{E}_{\text {eq }}$ is the local equivalent Green-Lagrange strain rate 5 and where $\dot{\bar{F}}_{x x}=\frac{\partial \bar{F}_{x x}}{\partial t}$ is the macroscopic deformation gradient rate along the $x$-direction. The value of $\dot{\bar{F}}_{x x}$ is controlled during the compression tests, while the other components of the macroscopic deformation gradient tensor $\overline{\mathbf{F}}$ follow in order to obtain a uniaxial homogenized state. It can be seen in Fig. 27 that the local equivalent strain rate has the same magnitude as the applied macroscopic one (with a maximal factor about 1.7) at the different levels of compressive

${ }^{5}$ The local equivalent Green-Lagrange strain rate is computed from

$$
\dot{E}_{\mathrm{eq}}=\frac{\partial E_{\mathrm{eq}}}{\partial t} \text { with } E_{e q}=\sqrt{\frac{2}{3} \operatorname{dev} \mathbf{E}^{\mathrm{GL}}: \operatorname{dev} \mathbf{E}^{\mathrm{GL}}} \text { and } \mathbf{E}^{\mathrm{GL}}=\frac{1}{2}\left(\mathbf{F}^{T} \cdot \mathbf{F}-\mathbf{I}\right) \text {, }
$$

where $\mathbf{F}$ is the local deformation gradient. 


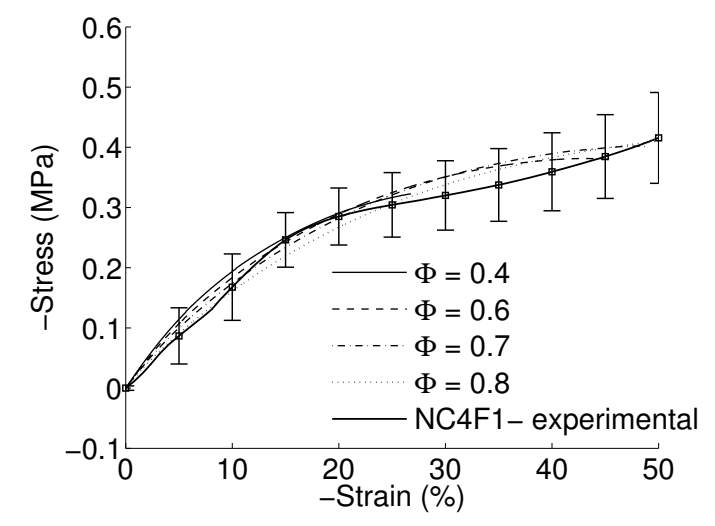

Figure 26: Compressive curves of the NC4F1 foam obtained experimentally and predicted by the the unit cell model with imperfections combination (Plateau border, square face curvature characterized by $\lambda=0.17$, and hexagonal face waviness characterized by $\theta=3$ and $\lambda=0.17)$.

strain - from $-5 \%$ to $-35 \%$. As the experimental tests are performed at low macroscopic strain-rates $(\simeq 0.0017 / \mathrm{s})$ and as the local equivalent strain rate remains close to the macroscopic one, the visco-elasticity effects could be neglected in the simulations.

\section{Conclusions}

The compressive properties of PP/CNTs nanocomposite foams were studied by both an experimental and a numerical multi-scale approaches.

The PP/CNTs nanocomposites were prepared by melt mixing and $\mathrm{ScCO}_{2}$ was used as foaming agent to prepare nanocomposite foams at different soaking temperatures. An increase of the soaking temperature led to an increase of the pore size and of the volume expansion ratio, and to a decrease of the cell density and of the relative density. DSC measurements were performed on both the un-foamed and foamed samples, and it was shown that the degree of crystallinity is reduced after the foaming process, yielding lower mechanical properties of the foam cell walls.

On the one hand, three-point bending tests were performed on nanocomposite (un-foamed) samples to measure the flexural modulus for different crystallinity levels. The values were found to depends on the degree of crystallinity. On the other hand, uniaxial compression tests were performed on foamed samples and different stress-strain behaviors were obtained due to 


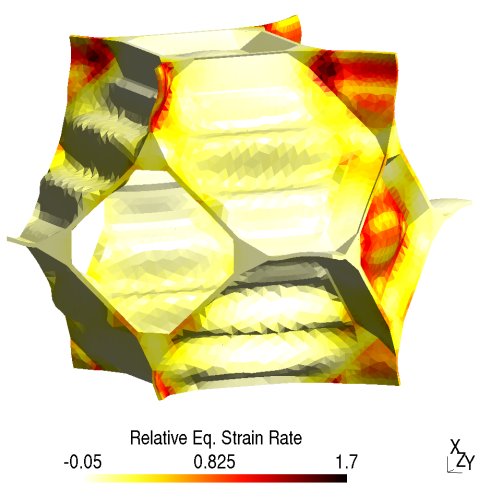

(a) $-5 \%$-strain

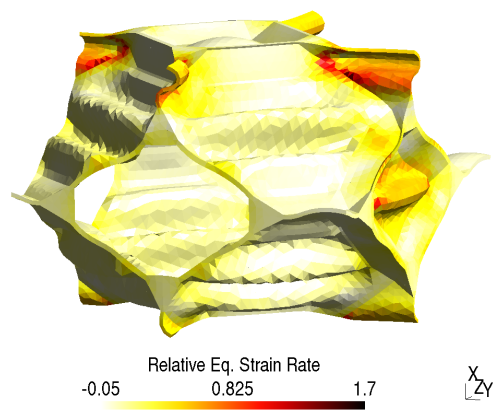

(c) $-25 \%$-strain

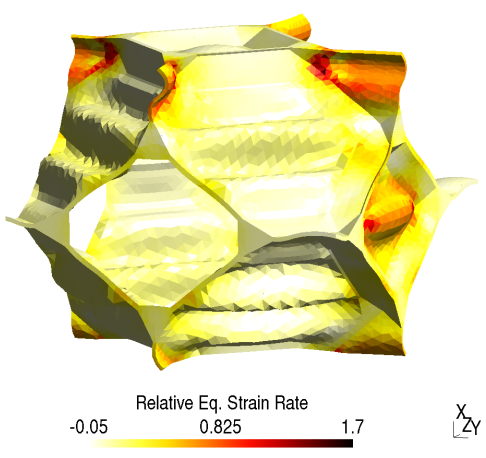

(b) $-15 \%$-strain

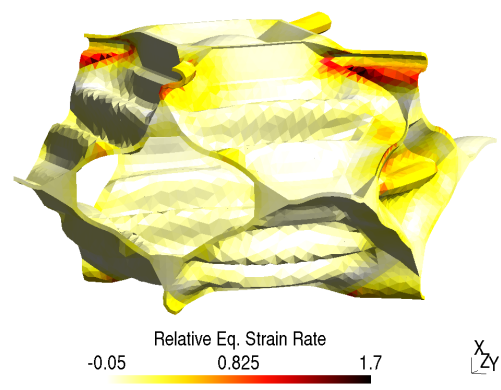

(d) $-35 \%$-strain

Figure 27: Distribution of the relative equivalent strain rate $\dot{\varepsilon}_{m}$ at different compressive macroscopic strains -(a) $-5 \%$, (b) $-15 \%$, (c) $-25 \%$, and (d) $-35 \%$ - for the NC4F1 foam modeled by the the unit cell with imperfections combination (Plateau border $\Phi=0.6$, square face curvature characterized by $\lambda=0.17$, and hexagonal face waviness characterized by $\theta=3$ and $\lambda=0.17)$.

the different micro-structure characteristics, micro-structure imperfections, and to the different degrees of crystallinity.

Since the cell wall thickness of the nanocomposite foams is of the $\mu \mathrm{m}$ order, direct measurements of their mechanical properties was not possible. Instead, a simple theoretical model, in which the Young's modulus of the cell walls is estimated from their degree of crystallinity and CNTs content, was proposed. The applicability of this model was demonstrated through experimental data, either provided in this paper or from the literature. The Young's modulus of the cell walls was then estimated from the measured 
degrees of crystallinity of the foamed samples.

A tetrakaidecahedron unit cell-based computational micro-mechanics model was then developed to estimate the homogenized properties of the nanocomposite foamed material. Several sources of imperfections were considered in the model: a mass concentration factor $\Phi$ characterizing the presence of more material in the cell junctions than in the center of the cell walls, a curvature of the cell face controlled by the shape factor $\lambda$, and a cell face waviness of period number $\theta$. The numerical results showed that the different parameters have an effect on the homogenized properties, mainly at low relative densities. The homogenized Young's modulus of the linear response was then estimated using this computational micro-mechanics model. It was shown that by considering the imperfections combined altogether, a value of $\Phi$ in between 0.4 and 0.8 gives a good prediction compared to the experiments for the $4 \% \mathrm{wt}$ and for the $8 \%$ wt CNTs foamed samples. Finally the overall non-linear compressive stress-strain curves of the foamed material were obtained by using a large strain elastic setting. The numerical prediction was shown to be in good agreement with the experimental data during the elastic response and the plateau stage.

In a future work, a more reliable geometry of the foam micro-structure will be obtained from tomographical images. A visco-elasto-plastic constitutive law can also be used to model the cell walls and self contact phenomena of the cell walls can be accounted for so that the overall stress-strain curve can be extended to the densification regime.

\section{Appendix A. Neo-Hookean hyperelastic constitutive model}

Under large deformations, the material constitutive model is based on the strain measure and on an elastic potential function of its work-conjugate stress. In case of a Neo-Hookean model, the deformation gradient $\mathbf{F}$ is chosen as the strain measure with its Jacobian $J=\operatorname{det} \mathbf{F}$ satisfying $J>0$. Its energetically conjugate stress is the first Piola-Kirchhoff stress $\mathbf{P}$, and the elastic potential is defined as

$$
U(J, \hat{\mathbf{C}})=\frac{K}{2} \log ^{2} J+\frac{\mu}{2}(\operatorname{tr}(\hat{\mathbf{C}})-3),
$$

where $K=\frac{E}{3(1-2 \nu)}$ and $\mu=\frac{E}{2(1+\nu)}$ are the bulk and shear moduli of the material, with the Young's modulus $E$ and the Poisson ratio $\nu$, and where 
$\hat{\mathbf{C}}=\mathbf{C} / J^{\frac{2}{3}}$, with the right Cauchy tensor $\mathbf{C}=\mathbf{F}^{T} \mathbf{F}$. Note that the defined elastic potential can only explicitly depends on the elastic deformation through the right Cauchy tensor C. The first Piola-Kirchhoff stress is thus given by

$$
\mathbf{P}=2 \mathbf{F} \frac{\partial U}{\partial \mathbf{C}}=\frac{\mu}{J^{\frac{2}{3}}}\left[\mathbf{F}-\frac{1}{3} \operatorname{tr}(\mathbf{C}) \mathbf{F}^{-T}\right]+K \log J \mathbf{F}^{-T},
$$

and the Cauchy stress can be computed by

$$
\boldsymbol{\sigma}=\frac{1}{J} \mathbf{P F}^{T}=\frac{\mu}{\mathrm{J}^{\frac{5}{3}}}\left[\mathbf{F}^{T} \mathbf{F}-\frac{1}{3} \operatorname{tr}(\mathbf{C}) \mathbf{I}\right]+K \log J \mathbf{I} .
$$

This material model can be compared in small deformation to a Hooke's law. Indeed, the small strain assumption leads to

$$
\begin{aligned}
& \mathbf{C}=\mathbf{F}^{T} \mathbf{F} \approx \mathbf{F F}^{T} \approx 2 \boldsymbol{\varepsilon}+\mathbf{I}, \\
& \log J \approx \operatorname{tr}(\boldsymbol{\varepsilon}), \text { and } \\
& J \approx 1 .
\end{aligned}
$$

where $\varepsilon$ is the small deformation tensor. Thus Eq. (A.3) reduces to

$$
\boldsymbol{\sigma}=\left(K-\frac{2}{3} \mu\right) \operatorname{tr}(\varepsilon) \mathbf{I}+2 \mu \varepsilon,
$$

that exactly expresses the Hooke's law in the case of elastic small strains.

\section{Acknowledgment}

Les recherches ont été financées grâce à la subvention "Actions de recherche concertées ARC 09/14-02 BRIDGING - From imaging to geometrical modelling of complex micro structured materials: Bridging computational engineering and material science" de la Direction générale de l'Enseignement non obligatoire de la Recherche scientifique, Direction de la Recherche scientifique, Communauté française de Belgique, et octroyées par l'Académie Universitaire Wallonie-Europe.

Christophe Detrembleur is Research Director by F.R.S.-FNRS and thanks the FNRS for its financial support. 


\section{References}

Affdl, J.C.H., Kardos, J.L., 1976. The Halpin-Tsai equations: a review. Polymer Engineering \& Science 16, 344-352.

Ameli, A., Nofar, M., Park, C., Ptschke, P., Rizvi, G., 2014. Polypropylene/carbon nanotube nano/microcellular structures with high dielectric permittivity, low dielectric loss, and low percolation threshold. Carbon 71, 206 - 217. URL: http://www.sciencedirect.com/science/ article/pii/S0008622314000670, doi:http://dx.doi.org/10.1016/j . carbon.2014.01.031.

Avalle, M., Belingardi, G., Ibba, A., 2007. Mechanical models of cellular solids: Parameters identification from experimental tests. International Journal of Impact Engineering 34, 3 - 27. URL: http:// WWw.sciencedirect.com/science/article/pii/S0734743X06001023, doi:http://dx.doi.org/10.1016/j.ijimpeng.2006.06.012 international Conference on Impact Loading of Lightweight Structures.

Bao, S., Tjong, S., 2008. Mechanical behaviors of polypropylene/carbon nanotube nanocomposites: The effects of loading rate and temperature. Materials Science and Engineering: A 485, 508 - 516. URL: http:// WWW.sciencedirect.com/science/article/pii/S0921509307016140, doi:http://dx.doi.org/10.1016/j.msea.2007.08.050.

Bédoui, F., Diani, J., Régnier, G., Seiler, W., 2006. Micromechanical modeling of isotropic elastic behavior of semicrystalline polymers. Acta Materialia 54, 1513 - 1523. URL: http://www.sciencedirect.com/science/ article/pii/S1359645405006920, doi:http://dx.doi.org/10.1016/j . actamat.2005.11.028.

Bikiaris, D., 2010. Microstructure and properties of polypropylene/carbon nanotube nanocomposites. Materials 3, 2884-2946. URL: http://www. mdpi.com/1996-1944/3/4/2884, doi:10.3390/ma3042884.

Chen, L., Schadler, L.S., Ozisik, R., 2011. An experimental and theoretical investigation of the compressive properties of multi-walled carbon nanotube/poly(methyl methacrylate) nanocomposite foams. Polymer 52, 2899 - 2909. URL: http://www.sciencedirect.com/science/article/ pii/S0032386111003557, doi:http://dx.doi.org/10.1016/j.polymer. 2011.04 .050 . 
Coleman, J.N., Khan, U., Blau, W.J., Gunko, Y.K., 2006. Small but strong: A review of the mechanical properties of carbon nanotubecpolymer composites. Carbon 44, 1624 - 1652. URL: http:// WWW.sciencedirect.com/science/article/pii/S0008622306001229, doi:http://dx.doi.org/10.1016/j.carbon.2006.02.038.

Czekanski, A., Elbestawi, M., Meguid, S., 2005. On the fe modeling of closed-cell aluminum foam. International Journal of Mechanics and Materials in Design 2, 23-34. URL: http://dx.doi.org/10.1007/ s10999-005-0518-7, doi:10.1007/s10999-005-0518-7.

Daxner, T., 2010. Finite Element Modeling of Cellular Materials. Springer Vienna. volume 521 of CISM International Centre for Mechanical Sciences. pp. 47-106. URL: http://dx.doi.org/10.1007/978-3-7091-0297-8_2, doi:10.1007/978-3-7091-0297-8\_2.

van Dommelen, J., Parks, D., Boyce, M., Brekelmans, W., Baaijens, F., 2003. Micromechanical modeling of the elasto-viscoplastic behavior of semi-crystalline polymers. Journal of the Mechanics and Physics of Solids 51, 519 - 541. URL: http://www. sciencedirect.com/science/article/pii/S0022509602000637, doi:http://dx.doi.org/10.1016/S0022-5096(02)00063-7.

Dondero, W.E., Gorga, R.E., 2006. Morphological and mechanical properties of carbon nanotube/polymer composites via melt compounding. Journal of Polymer Science Part B: Polymer Physics 44, 864-878. URL: http: //dx.doi.org/10.1002/polb.20743, doi:10.1002/polb.20743.

Drozdov, A., Gupta, R., 2003. Non-linear viscoelasticity and viscoplasticity of isotactic polypropylene. International Journal of Engineering Science 41, 2335 - 2361. URL: http://www . sciencedirect.com/science/article/pii/S0020722503002398, doi:http://dx.doi.org/10.1016/S0020-7225(03)00239-8.

Gan $\beta$, M., Satapathy, B.K., Thunga, M., Weidisch, R., Pötschke, P., Jehnichen, D., 2008. Structural interpretations of deformation and fracture behavior of polypropylene/multi-walled carbon nanotube composites. Acta Materialia 56, 2247 - 2261. URL: http:// WwW.sciencedirect.com/science/article/pii/S1359645408000451, doi:http://dx.doi.org/10.1016/j.actamat.2008.01.010. 
Gibson, L.J., Ashby, M.F., 1997. Cellular solids: structure and properties (2nd ed). Cambridge University Press.

Grenestedt, J., 2005. On interactions between imperfections in cellular solids. Journal of Materials Science 40, 5853-5857. URL: http://dx.doi.org/ 10.1007/s10853-005-5019-4, doi:10.1007/s10853-005-5019-4.

Ibeh, C.C., Bubacz, M., 2008. Current trends in nanocomposite foams. Journal of Cellular Plastics 44, 493-515. URL: http://cel.sagepub. com/content/44/6/493.abstract, doi:10.1177/0021955X08097707, arXiv:http://cel.sagepub.com/content/44/6/493.full.pdf+html.

Jose, M.V., Dean, D., Tyner, J., Price, G., Nyairo, E., 2007. Polypropylene/carbon nanotube nanocomposite fibers: Process-morphologyproperty relationships. Journal of Applied Polymer Science 103, 38443850. URL: http://dx.doi.org/10.1002/app.25475, doi:10.1002/app. 25475.

Kang, C.H., Yoon, K.H., Park, Y.B., Lee, D.Y., Jeong, S.S., 2010. Properties of polypropylene composites containing aluminum/multi-walled carbon nanotubes. Composites Part A: Applied Science and Manufacturing 41, 919 - 926. URL: http://www.sciencedirect.com/science/ article/pii/S1359835X10000990, doi:http://dx.doi.org/10.1016/j . compositesa.2010.03.011.

Kanit, T., Forest, S., Galliet, I., Mounoury, V., Jeulin, D., 2003. Determination of the size of the representative volume element for random composites: statistical and numerical approach. International Journal of Solids and Structures 40, 3647-3679. URL: http://www.sciencedirect.com/science/article/ B6VJS-48GDV9K-1/2/2dd6cda9c49706ed2c969bdea531bb50, doi:DOI : 10 . 1016/S0020-7683(03)00143-4.

Lavengood, R., Goettler, L., 1971. Stiffness of non-aligned fiber reinforced composites. Technical Report.

Lee, L.J., Zeng, C., Cao, X., Han, X., Shen, J., Xu, G., 2005. Polymer nanocomposite foams. Composites Science and Technology 65, 2344 - 2363. URL: http://www.sciencedirect.com/science/article/pii/ 
S0266353805002253, doi/http://dx.doi.org/10.1016/j.compscitech. 2005.06.016. 20th Anniversary Special Issue.

Leelapornpisit, W., Ton-That, M.T., Perrin-Sarazin, F., Cole, K.C., Denault, J., Simard, B., 2005. Effect of carbon nanotubes on the crystallization and properties of polypropylene. Journal of Polymer Science Part B: Polymer Physics 43, 2445-2453. URL: http://dx.doi.org/10.1002/polb.20527, doi:10.1002/polb.20527.

López, J., Mutjé, P., Angels Pèlach, M., El Mansouri, N.E., Boufi, S., Vilaseca, F., 2012. Analysis of the tensile modulus of polypropylene composites reinforced with stone groundwood fibers. BioResources 7, 1310-1323. URL: http://www.scopus.com/ inward/record. url?eid=2-s2.0-84859082769\&partnerID=40\&md5= 5e77d7570b80f58263615d55a4b443c4.

Manchado, M.L., Valentini, L., Biagiotti, J., Kenny, J., 2005. Thermal and mechanical properties of single-walled carbon nanotubespolypropylene composites prepared by melt processing. Carbon 43, 1499 - 1505. URL: http://www.sciencedirect.com/science/article/pii/ S0008622305000771, doi:http://dx.doi.org/10.1016/j.carbon. 2005. 01.031 .

McIntosh, D., Khabashesku, V.N., Barrera, E.V., 2006. Nanocomposite fiber systems processed from fluorinated single-walled carbon nanotubes and a polypropylene matrix. Chemistry of Materials 18, 4561-4569. URL: http://pubs.acs. org/doi/abs/10.1021/cm060513q, doi 10.1021/cm060513q, arXiv:http://pubs.acs.org/doi/pdf/10.1021/cm060513q.

Meguid, S., Cheon, S., El-Abbasi, N., 2002. $\{\mathrm{FE}\}$ modelling of deformation localization in metallic foams. Finite Elements in Analysis and Design 38, 631 - 643. URL: http://www. sciencedirect.com/science/article/pii/S0168874X01000968, doi:http://dx.doi.org/10.1016/S0168-874X (01)00096-8.

Mortazavian, S., Fatemi, A., 2015. Effects of fiber orientation and anisotropy on tensile strength and elastic modulus of short fiber reinforced polymer composites. Composites Part B: Engineering 72, 116 - 129. URL: http://www.sciencedirect.com/science/article/pii/ 
S1359836814005642, doi:http://dx.doi.org/10.1016/j.compositesb. 2014.11.041.

Nguyen, V.D., Béchet, E., Geuzaine, C., Noels, L., 2012. Imposing periodic boundary condition on arbitrary meshes by polynomial interpolation. Computational Materials Science 55, 390 406. URL: http://wwW.sciencedirect.com/science/article/pii/ S0927025611005866, doi:10.1016/j.commatsci.2011.10.017.

Nguyen, V.D., Noels, L., 2014. Computational homogenization of cellular materials. International Journal of Solids and Structures 51, 2183 - 2203. URL: http://www.sciencedirect.com/science/ article/pii/S0020768314000778, doi:http://dx.doi.org/10.1016/j . ijsolstr.2014.02.029.

Nikolov, S., Doghri, I., Pierard, O., Zealouk, L., Goldberg, A., 2002. Multiscale constitutive modeling of the small deformations of semi-crystalline polymers. Journal of the Mechanics and Physics of Solids 50, 2275 - 2302. URL: http://www.sciencedirect.com/science/article/pii/ S0022509602000364, doi:http://dx.doi.org/10.1016/S0022-5096(02) 00036-4.

Parenteau, T., Ausias, G., Grohens, Y., Pilvin, P., 2012. Structure, mechanical properties and modelling of polypropylene for different degrees of crystallinity. Polymer 53, 5873 - 5884. URL: http:// WWW.sciencedirect.com/science/article/pii/S0032386112008385, doi:http://dx.doi.org/10.1016/j.polymer.2012.09.053.

Pedrazzoli, D., Pegoretti, A., 2014. Hybridization of short glass fiber polypropylene composites with nanosilica and graphite nanoplatelets. Journal of Reinforced Plastics and Composites 33, 1682-1695. URL: http://jrp.sagepub.com/ content/33/18/1682.abstract, doi:10.1177/0731684414542668, arXiv:http://jrp.sagepub.com/content/33/18/1682.full.pdf+html.

Peric, D., de Souza Neto, E.A., Feijóo, R.A., Partovi, M., Molina, A.J.C., 2010. On micro-to-macro transitions for multi-scale analysis of non-linear heterogeneous materials: unified variational basis and finite element implementation. Int. J. Numer. Meth. Engng. URL: http://dx.doi.org/ 10.1002/nme.3014. 
Prashantha, K., Soulestin, J., Lacrampe, M., Krawczak, P., Dupin, G., Claes, M., 2009. Masterbatch-based multi-walled carbon nanotube filled polypropylene nanocomposites: Assessment of rheological and mechanical properties. Composites Science and Technology 69, 1756 1763. URL: http://www.sciencedirect.com/science/article/pii/ S0266353808004089, doi:http://dx.doi.org/10.1016/j.compscitech. 2008.10.005. experimental Techniques and Design in Composite Materials (ETDCM8) with Regular Papers.

Reitz, D., Schuetz, M., Glicksman, L., 1984. A basic study of aging of foam insulation. Journal of Cellular Plastics 20, 104-113. URL: http://cel.sagepub.com/ content/20/2/104.short doi:10.1177/0021955X8402000202, arXiv:http://cel. sagepub.com/content/20/2/104.full.pdf+html.

Ryu, K.M., An, J.Y., Cho, W.S., Yoo, Y.C., Kim, H.S., 2005. Mechanical modeling of al-mg alloy open-cell foams. Materials Transactions 46, 622625. doi 10.2320 /matertrans.46.622.

Santosa, S., Wierzbicki, T., 1998. On the modeling of crush behavior of a closed-cell aluminum foam structure. Journal of the Mechanics and Physics of Solids 46, 645 - 669. URL: http:// www.sciencedirect.com/science/article/pii/S0022509697000823, doi:http://dx.doi.org/10.1016/S0022-5096(97)00082-3.

Seidel, G.D., Lagoudas, D.C., 2006. Micromechanical analysis of the effective elastic properties of carbon nanotube reinforced composites. Mechanics of Materials 38, 884 - 907. URL: http://www. sciencedirect.com/science/article/pii/S0167663605001699, doi:http://dx.doi.org/10.1016/j.mechmat.2005.06.029. advances in Disordered Materials.

Serrano, A., Espinach, F., Tresserras, J., del Rey, R., Pellicer, N., Mutje, P., 2014. Macro and micromechanics analysis of short fiber composites stiffness: The case of old newspaper fiberscpolypropylene composites. Materials \& Design 55, 319 324. URL: http://wWw.sciencedirect.com/science/article/pii/ S0261306913009382, doi:http://dx.doi.org/10.1016/j.matdes.2013. 10.011 . 
Simone, A., Gibson, L., 1998a. Effects of solid distribution on the stiffness and strength of metallic foams. Acta Materialia 46, 2139 2150. URL: http://wwW.sciencedirect.com/science/article/pii/ S1359645497004217, doi:http://dx.doi.org/10.1016/S1359-6454(97) 00421-7.

Simone, E., Gibson, L., 1998b. The effects of cell face curvature and corrugations on the stiffness and strength of metallic foams. Acta Mater. 46, 3929-3935. URL: http://linkinghub.elsevier.com/retrieve/pii/ S135964549800072X, doi:10.1016/S1359-6454(98)00072-X.

Soitong, T., Pumchusak, J., 2011. The relationship of crystallization behavior, mechanical properties, and morphology of polypropylene nanocomposite fibers. Journal of Materials Science 46, 1697-1704. URL: http://dx.doi.org/10.1007/s10853-010-4987-1, doi:10.1007/ s10853-010-4987-1.

Spieckermann, F., Wilhelm, H., Kerber, M., Schafler, E., Polt, G., Bernstorff, S., Addiego, F., Zehetbauer, M., 2010. Determination of lamella thickness distributions in isotactic polypropylene by X-ray line profile analysis. Polymer 51, 4195 - 4199. URL: http://www.sciencedirect.com/science/ article/pii/S0032386110006087, doi:http://dx.doi.org/10.1016/j. polymer.2010.07.009.

Spitalsky, Z., Tasis, D., Papagelis, K., Galiotis, C., 2010. Carbon nanotube-polymer composites: Chemistry, processing, mechanical and electrical properties. Progress in Polymer Science 35, 357 - 401. URL: http://wWw.sciencedirect.com/science/ article/pii/S0079670009000859, doi:http://dx.doi.org/10.1016/j. progpolymsci.2009.09.003.

Stan, F., Sandu, L.I., Fetecau, C., 2014. Effect of processing parameters and strain rate on mechanical properties of carbon nanotubes filled polypropylene nanocomposites. Composites Part B: Engineering 59, 109 - 122. URL: http://www.sciencedirect.com/science/article/pii/ S1359836813006975, doi:http://dx.doi.org/10.1016/j.compositesb. 2013.11.023.

Takada, M., Tanigaki, M., Ohshima, M., 2001. Effects of co2 on crystallization kinetics of polypropylene. Polymer Engineering \& Science 41, 1938- 
1946. URL: http://dx.doi.org/10.1002/pen.10890, doi:10.1002/pen. 10890.

Thomassin, J.M., Huynen, I., Jerome, R., Detrembleur, C., 2010. Functionalized polypropylenes as efficient dispersing agents for carbon nanotubes in a polypropylene matrix; application to electromagnetic interference (emi) absorber materials. Polymer 51, 115 - 121. URL: http://www.sciencedirect.com/science/article/ pii/S0032386109009793, doi:http://dx.doi.org/10.1016/j.polymer. 2009.11.012.

Tran, M.P., Detrembleur, C., Alexandre, M., Jerome, C., Thomassin, J.M., 2013. The influence of foam morphology of multi-walled carbon nanotubes/poly(methyl methacrylate) nanocomposites on electrical conductivity. Polymer 54, 3261 - 3270. URL: http:// www.sciencedirect.com/science/article/pii/S0032386113002723, doi:http://dx.doi.org/10.1016/j.polymer.2013.03.053.

Tsai, S.W., Pagano, N.J., 1968. Invariant properties of composite materials. Technical Report.

de Villoria, R.G., Miravete, A., 2007. Mechanical model to evaluate the effect of the dispersion in nanocomposites. Acta Materialia 55, 3025 - 3031. URL: http://www.sciencedirect.com/science/article/ pii/S1359645407000602, doi:http://dx.doi.org/10.1016/j.actamat. 2007.01.007.

van der Wal, A., Mulder, J., Gaymans, R., 1998. Fracture of polypropylene: The effect of crystallinity. Polymer 39, 5477 5481. URL: http://www.sciencedirect.com/science/article/pii/ S0032386197102798, doi:http://dx.doi.org/10.1016/S0032-3861(97) 10279-8.

Weon, J.I., Sue, H.J., 2006. Mechanical properties of talc- and caco3reinforced high-crystallinity polypropylene composites. Journal of Materials Science 41, 2291-2300. URL: http://dx.doi.org/10.1007/ s10853-006-7171-x, doi:10.1007/s10853-006-7171-x.

Xia, H., Wang, Q., Li, K., Hu, G.H., 2004. Preparation of polypropylene/carbon nanotube composite powder with a solid-state mechanochem- 
ical pulverization process. Journal of Applied Polymer Science 93, 378386. URL: http://dx.doi.org/10.1002/app.20435, doi:10.1002/app. 20435 .

Youssef, S., Maire, E., Gaertner, R., 2005. Finite element modelling of the actual structure of cellular materials determined by x-ray tomography. Acta Materialia 53, 719 - 730. URL: http://www. sciencedirect.com/science/article/pii/S1359645404006330, doi:http://dx.doi.org/10.1016/j.actamat.2004.10.024.

Zhang, J., Kikuchi, N., Li, V., Yee, A., Nusholtz, G., 1998. Constitutive modeling of polymeric foam material subjected to dynamic crash loading. International Journal of Impact Engineering 21, 369 - 386. URL: http://www.sciencedirect.com/science/article/pii/ S0734743X97000870, doi:http://dx.doi.org/10.1016/S0734-743X (97) 00087-0.

Zhu, H., Knott, J., Mills, N., 1997. Analysis of the elastic properties of open-cell foams with tetrakaidecahedral cells. Journal of the Mechanics and Physics of Solids 45, 319 - 343. URL: http:// WwW.sciencedirect.com/science/article/pii/S0022509696000907. doi:http://dx.doi.org/10.1016/S0022-5096(96)00090-7.

Zrida, M., Laurent, H., Rio, G., Pimbert, S., Grolleau, V., Masmoudi, N., Bradai, C., 2009. Experimental and numerical study of polypropylene behavior using an hyper-visco-hysteresis constitutive law. Computational Materials Science 45, 516 - 527. URL: http:// WWW.sciencedirect.com/science/article/pii/S0927025608005028, doi:http://dx.doi.org/10.1016/j.commatsci.2008.11.017. 